\title{
Gas and dust cooling along the major axis of M 33 (HerM33es) ISO/LWS [C II] observations ${ }^{\star, \star \star ~}$
}

\author{
C. Kramer $^{1}$, J. Abreu-Vicente ${ }^{1}$, S. García-Burillo² , M. Relaño ${ }^{3}$, S. Aalto ${ }^{4}$, M. Boquien ${ }^{5}$, J. Braine ${ }^{6}$, C. Buchbender ${ }^{1}$, \\ P. Gratier ${ }^{6,7}$, F. P. Israel ${ }^{8}$, T. Nikola ${ }^{9}$, M. Röllig ${ }^{10}$, S. Verley ${ }^{3}$, P. van der Werf ${ }^{8}$, and E. M. Xilouris ${ }^{11}$ \\ ${ }^{1}$ Instituto Radioastronomía Milimétrica (IRAM), Av. Divina Pastora 7, Nucleo Central, 18012 Granada, Spain \\ e-mail: kramer@iram.es \\ 2 Observatorio Astronómico Nacional (OAN) - Observatorio de Madrid, Alfonso XII 3, 28014 Madrid, Spain \\ 3 Departamento de Fisica Teórica y del Cosmos, Universidad de Granada, 18071 Granada, Spain \\ ${ }^{4}$ Department of Radio and Space Science, Onsala Observatory, Chalmers University of Technology, 43992 Onsala, Sweden \\ 5 Aix Marseille Université, CNRS, LAM (Laboratoire d'Astrophysique de Marseille) UMR 7326, 13388 Marseille, France \\ ${ }^{6}$ Laboratoire d'Astrophysique de Bordeaux, Observatoire de Bordeaux, 33270 Floirac, France \\ 7 IRAM, 300 rue de la Piscine, 38406 Saint-Martin d'Hères, France \\ ${ }^{8}$ Leiden Observatory, Leiden University, PO Box 9513, 2300 RA Leiden, The Netherlands \\ 9 Department of Astronomy, Cornell University, Ithaca, NY 14853, USA \\ 10 KOSMA, I. Physikalisches Institut, Universität zu Köln, Zülpicher Straße 77, 50937 Köln, Germany \\ 11 Institute of Astronomy and Astrophysics, National Observatory of Athens, P. Penteli, 15236 Athens, Greece
}

Received 1 November 2012 / Accepted 29 March 2013

\begin{abstract}
Aims. We aim to better understand the heating of gas by observing the prominent gas cooling line [C II] at $158 \mu \mathrm{m}$ in the lowmetallicity environment of the Local Group spiral galaxy M33 on scales of $280 \mathrm{pc}$. In particular, we describe the variation of the photoelectric heating efficiency with the galactic environment.

Methods. In this study, we present [C II] observations along the major axis of M33 using the Infrared Space Observatory in combination with Herschel continuum maps, IRAM $30 \mathrm{~m} \mathrm{CO} \mathrm{2-1,} \mathrm{and} \mathrm{VLA} \mathrm{H} \mathrm{I} \mathrm{data} \mathrm{to} \mathrm{study} \mathrm{the} \mathrm{variation} \mathrm{in} \mathrm{velocity} \mathrm{integrated} \mathrm{intensities.}$ The ratio of [C II] emission over the far-infrared continuum is used as a proxy for the heating efficiency, and models of photondominated regions are used to study the local physical densities, far-ultraviolet radiation fields, and average column densities of the molecular clouds.

Results. The heating efficiency stays constant at $0.8 \%$ in the inner $4.5 \mathrm{kpc}$ radius of the galaxy, where it increases to reach values of $\sim 3 \%$ in the outskirts at about a $6 \mathrm{kpc}$ radial distance. The rise of efficiency is explained in the framework of PDR models by lowered volume densities and FUV fields for optical extinctions of only a few magnitudes at constant metallicity. For the significant fraction of H I emission stemming from PDRs and for typical pressures found in the Galactic cold neutral medium (CNM) traced by $\mathrm{HI}$ emission, the CNM contributes $\sim 15 \%$ to the observed [C II] emission in the inner $2 \mathrm{kpc}$ radius of M 33 . The CNM contribution remains largely undetermined in the south, while positions between radial distances of 2 and $7.3 \mathrm{kpc}$ in the north of M 33 show a contribution of $\sim 40 \% \pm 20 \%$.
\end{abstract}

Key words. galaxies: ISM - photon-dominated region (PDR) - ISM: structure - evolution

\section{Introduction}

In photon-dominated regions (PDRs), far-ultraviolet (FUV) photons from stars dominate the chemistry and the energy balance in the interstellar gas. All the atomic and a large part of the molecular hydrogen of the interstellar medium (ISM) are located in PDRs, which emit a large fraction of far infrared (FIR) and millimeter emission (Tielens \& Hollenbach 1985; Bakes \& Tielens 1994; Hollenbach \& Tielens 1997).

The [C II] FIR fine structure line at $157.7 \mu \mathrm{m}$ is the most important gas coolant. The [O I] $63 \mu \mathrm{m}$ fine structure line starts to dominate in denser and warmer regions, when densities exceed

\footnotetext{
* Herschel is an ESA space observatory with science instruments provided by European-led PrincipalInvestigator consortia and with important participation from NASA.

$\star \star$ Appendices are available in electronic form at http://www . aanda.org
}

about $10^{4} \mathrm{~cm}^{-3}$ (Röllig et al. 2006). The photoelectric effect provides one of the dominant gas heating processes in PDRs. FUV photons eject electrons from dust grains or polycyclic aromatic hydrocarbon (PAH) molecules, heating the gas with their kinetic energy. Theoretical models have predicted efficiencies $\epsilon_{\mathrm{PE}}$ of up to a few percent (Weingartner \& Draine 2001), consistent with observations. The ratio of emerging [C II] intensity over the infrared continuum radiated by the dust has often been used as a measure of this efficiency. Observations of clouds in the Milky Way show variations over more than 2 orders of magnitude, between $10^{-4}$ and $3 \times 10^{-2}$ (e.g. Vastel et al. 2001; Habart et al. 2001; Mizutani et al. 2004; Jakob et al. 2007). A similar variation is found in observations of external galaxies (e.g. Malhotra et al. 2001; Rubin et al. 2009). The scatter has been attributed to changes in the mean charge of small grains and PAHs (Okada et al. 2013). However, the change of $\epsilon_{\mathrm{PE}}$ in low-metallicity environments, such as those encountered in the 
Table 1. Observation Log of all [C II] observations of ISO/LWS along the major axis of M33.

\begin{tabular}{|c|c|c|c|c|c|c|c|c|}
\hline IDA name & Abbrev. $^{a}$ & $\mathrm{TDT}^{b}$ (Obs. \#ID) & $\mathrm{RA}^{c}$ & Dec. $^{c}$ & Observer ID & Ref. & Type & AOT \\
\hline M 33S2 & S2 & 59901107 & $01 \mathrm{~h} 33 \mathrm{~m} 08.5 \mathrm{~s}$ & $+30 \mathrm{~d} 17 \mathrm{~m} 00.0 \mathrm{~s}$ & KMOCHIZU & - & Raster & $\mathrm{LO} 2$ \\
\hline M 33S & S1 & 78600403 & $01 \mathrm{~h} 33 \mathrm{~m} 37.1 \mathrm{~s}$ & $+30 \mathrm{~d} 31 \mathrm{~m} 34.7 \mathrm{~s}$ & KMOCHIZU & (1) & Raster & $\mathrm{LO} 2$ \\
\hline M 33 Nucleus & & 80800367 & $01 \mathrm{~h} 33 \mathrm{~m} 50.9 \mathrm{~s}$ & $+30 \mathrm{~d} 39 \mathrm{~m} 36.8 \mathrm{~s}$ & HSMITH & $(1,2)$ & Point & $\mathrm{LO} 2$ \\
\hline M $33 \mathrm{~N}$ & N1 & 78600801 & $01 \mathrm{~h} 34 \mathrm{~m} 07.3 \mathrm{~s}$ & $+30 \mathrm{~d} 46 \mathrm{~m} 55.7 \mathrm{~s}$ & KMOCHIZU & - & Raster & $\mathrm{LO} 2$ \\
\hline M 33N2 & $\mathrm{N} 2$ & 59900605 & $01 \mathrm{~h} 34 \mathrm{~m} 36.3 \mathrm{~s}$ & $+31 \mathrm{~d} 01 \mathrm{~m} 29.2 \mathrm{~s}$ & KMOCHIZU & - & Raster & $\mathrm{LO} 2$ \\
\hline
\end{tabular}

Notes. ${ }^{(a)}$ Abbreviation used in the text. ${ }^{(b)}$ Target Dedicated Time. ${ }^{(c)}$ The given coordinates are the central position of each raster strip of 19 positions.

References. (1) Brauher et al. (2008); (2) Higdon et al. (2003).

Magellanic Clouds or M33, is not yet well understood (e.g. Israel \& Maloney 2011). Interestingly, the efficiency drops for local ultra-luminous infrared galaxies (ULIRGs; Luhman et al. 2003; Graciá-Carpio et al. 2011) and for some ULIRGs at high redshifts (e.g. Stacey et al. 2010; Cox et al. 2011).

M33 is a nearby galaxy located at $840 \mathrm{kpc}$ distance (Freedman et al. 1991). Its overall metallicity is about half-solar (Magrini et al. 2010), which is only slightly higher than that of the Large Magellanic Cloud (LMC; Hunter et al. 2007). M 33 is an Sc galaxy which exhibits a prominent, flocculent spiral structure together with an underlying extended diffuse component. This structure is seen in the $250 \mu \mathrm{m}$ map of M 33 which was observed in the framework of the Herschel open time key project HerM33es (Kramer et al. 2010) (Fig. 1). M33 has a moderate inclination of $56^{\circ}$, which allows studies of the ISM at a low depth along the line-of-sight. Its proximity allows high spatial resolution studies.

While previous studies have only discussed [C II] emission at a few selected positions in M 33, there have been no systematic studies that describe the spatial variation of [C II] emission in the disk of the galaxy. Higdon et al. (2003) used ISO/LWS ${ }^{1}$ to study [C II] other FIR emission lines, and the continuum in the nucleus and in six H II regions. They found $[\mathrm{CII}] / \mathrm{FIR}_{\mathrm{LWS}}$ values between $0.2 \%$ and $0.7 \%$. Brauher et al. (2008) compiled ISO/LWS data of 227 galaxies, which included 23 positions in M 33 with [C II $]$ data. Plotting the $[\mathrm{CII}] /$ FIR ratio for all galaxies, they find variations from $10^{-4}$ to greater than $1 \%$. Mookerjea et al. (2011) and Braine et al. (2012) analyzed the first Herschel/PACS and HIFI spectroscopic data sets of the HerM33es project. They found $[\mathrm{C} \mathrm{II}] /$ FIR ratios between $0.01 \%$ and $2 \%$ in a $2^{\prime} \times 2^{\prime}$ box centered on the BCLMP $302 \mathrm{H}$ II region, and a ratio of $1.1 \%$ at the position of the BCLMP $691 \mathrm{H}$ II region that lies at galactocentric distances of 2.1 and $3.3 \mathrm{kpc}$, respectively, along the major axis.

In this paper, we present archival ISO/LWS [C II] data along the major axis of M 33 up to a galacto-centric distance of $8 \mathrm{kpc}$. We study the radial distributions and correlations among [C II], the FIR continuum, $\mathrm{CO}, \mathrm{HI}$, and $\mathrm{H} \alpha$. We also study the radial distribution of the $[\mathrm{C} \mathrm{II}] /$ FIR ratio. We compare the observations in M33 with data of star-forming regions in the Milky Way with other external galaxies including low-metallicity objects. Local volume densities and FUV fields of the [C II] and CO emitting gas are estimated using the Kaufman et al. (1999, K99) PDR model. The observed [C II] emission is also compared with an estimate of the [C II] emission emitted by atomic clouds.

\footnotetext{
1 Infrared Space Observatory (Kessler et al. 1996)/Long-Wave Spectrometer (Gry et al. 2003).
}

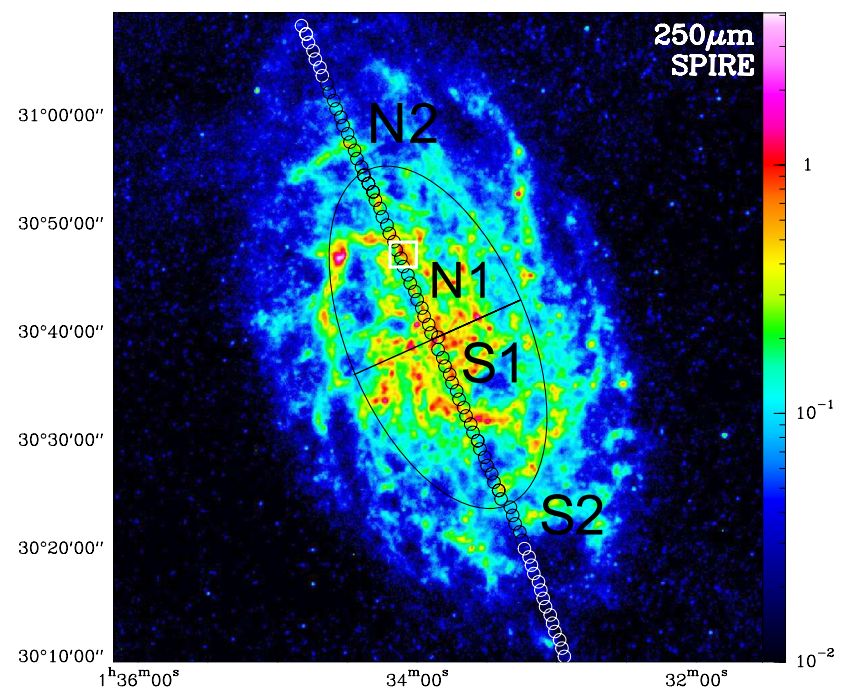

Fig. 1. Herschel SPIRE $250 \mu \mathrm{m}$ map of M33 (Xilouris et al. 2012). Units are $\mathrm{Jy} /\left(18^{\prime \prime}\right.$ beam $)$. Circles mark the position and beam size of the ISO/LWS [C II] observations along the major axis of M 33. The ellipse delineates the galacto-centric distance of $4.5 \mathrm{kpc}$, which devides the observed inner (N1 and S1) and outer (N2 and S2) regions. The white square marks the BCLMP $302 \mathrm{H}$ II region. Coordinates are RA and Dec. (Eq. J2000).

\section{Observations and data analysis}

\section{1. [C II] $158 \mu \mathrm{m}$ (ISO/LWS)}

We list all ISO/LWS [C II] spectra observed along the major axis of M 33 at a position angle of $23^{\circ}$ in Table 1 . The spectra were observed using the partial grating scan mode (LWS AOT ${ }^{2}$ LO2). This AOT covers the wavelength range 43-196.9 $\mu \mathrm{m}$, and it has a medium spectral resolution of $\Delta \lambda / \lambda \sim 200$ which corresponds to $1500 \mathrm{~km} \mathrm{~s}^{-1}$ at the wavelength of the [C II] line. The LWS flux-calibration and its relative spectral response function were derived from observations of Uranus (Swinyard et al. 1998). The angular resolution is 69.4" (Gry et al. 2003) which corresponds to a linear resolution of $280 \mathrm{pc}$. The spectra have been automatically processed by the ISO system. We retrieved spectra at 77 positions from the ISO Data Archive (IDA) for further processing. The observed positions cover about $\pm 8 \mathrm{kpc}\left( \pm 33^{\prime}\right)$ from the nucleus on a grid of about $208 \mathrm{pc}$ (Fig. 1).

We averaged the observations at each position, subtracted linear baselines, and fitted a Gaussian to the line. Data were analyzed using the ISO Spectral Analysis Package (ISAP v2.1 Sturm et al. 1998). We assume a calibration error of $15 \%$

\footnotetext{
2 Astronomical Observing Template.
} 
(Higdon et al. 2003). Some sample spectra are shown in Appendix C.

Figure 2 shows the variation of $[\mathrm{C}$ II] intensities along the major axis. [C II] is detected above $3 \sigma$ at 36 positions. Stacking of the neighboring positions increased the number of detections. Tables A. 1 and A. 2 in the Appendix list the stacked positions.

\subsection{Far-infrared continuum}

To measure the total FIR continuum, we combined SPIRE and PACS maps of M33 at five wavelengths between 500 and $100 \mu \mathrm{m}$ (Boquien et al. 2011; Xilouris et al. 2012), which are taken in the framework of HerM33es with MIPS/Spitzer 24 and $70 \mu \mathrm{m}$ maps (Verley et al. 2007; Tabatabaei et al. 2007). These maps were smoothed to the ISO/LWS resolution using Gaussian kernels. The fluxes were extracted using circular apertures of $69.4^{\prime \prime}$ centered at the ISO/LWS positions. A twocomponent greybody function was fitted to the spectral energy distribution (SED) at each position, following the method described in Kramer et al. (2010). Integrating between $42.5 \mu \mathrm{m}$ and $122.5 \mu \mathrm{m}$ (Dale \& Helou 2002) yields the FIR surface brightness. The dust emissivity index $\beta$ was fixed at 1.5 , which was found to be the best-fitting value for M33 (Kramer et al. 2010; Xilouris et al. 2012). The total FIR luminosity is, however, robust against changes of $\beta$. Figure 2 shows the variation of relative FIR intensities along the major axis (cf. Table B.1). A few sample SEDs are shown in Appendix C. We also integrated the fitted SEDs over the range 3-1000 $\mu \mathrm{m}$ wavelengths to estimate total infrared (TIR) intensities (Dale \& Helou 2002), thereby deriving the ratios between TIR over FIR, which lie between 2.7 and 1.3 (Table B.1).

\section{3. $\mathrm{CO}, \mathrm{HI}, \mathrm{H} \alpha$}

Complementary $\mathrm{CO}$ and $\mathrm{HI}$ data were used as tracers of the molecular gas and the atomic gas to compare with [C II] emission. The CO 2-1 line was mapped with the IRAM 30m telescope by Gardan et al. (2007) and Gratier et al. (2010). These maps cover the major axis up to a distance of $8.5 \mathrm{kpc}$ in the north and $6.5 \mathrm{kpc}$ in the south. The CO map covers all ISO/LWS [C II] positions, except for the eight southernmost positions.

We determined $3 \sigma$ upper limits of the integrated intensities using $\sigma=\sqrt{N} \Delta v_{\text {res }} T_{\mathrm{mb}}^{\mathrm{rms}}$ where the number of channels is defined as $N$ over the velocity extent of the line, the velocity resolution $\Delta v_{\text {res }}$, and corresponding baseline rms $T_{\mathrm{mb}}^{\mathrm{rms}}$. The H I VLA map of M 33 (Gratier et al. 2010) covers the entire galaxy up to $8.5 \mathrm{kpc}$ radial distance. $\mathrm{H} \mathrm{I}$ is detected at all ISO/LWS positions. While no single dish data were combined with the interferometric observations, the total flux recovered over the entire galaxy by the interferometric observations alone corresponds to more than $90 \%$ of the flux measured at the Arecibo single dish telescope (Putman et al. 2009).

We also used a map of $\mathrm{H} \alpha$ emission presented in Hoopes \& Walterbos (2000) and by Verley et al. (2007). These data were obtained at the $0.6 \mathrm{~m}$ Burrell-Schmidt telescope at Kitt Peak National Observatory (KPNO). $\mathrm{H} \alpha$ is detected at $42 \mathrm{ISO} / \mathrm{LWS}$ positions.

Intensities have been calculated by smoothing all data to the angular resolution of the LWS [C II] data, as described in the Appendix B. The variation of relative intensities of $\mathrm{CO}, \mathrm{HI}$, and $\mathrm{H} \alpha$ is shown in Fig. 2, and absolute intensities are listed in Table B.1. We stacked $\mathrm{CO}, \mathrm{HI}$, and $\mathrm{H} \alpha$ over the same positions as [C II] (cf. Tables A.1, A.2).
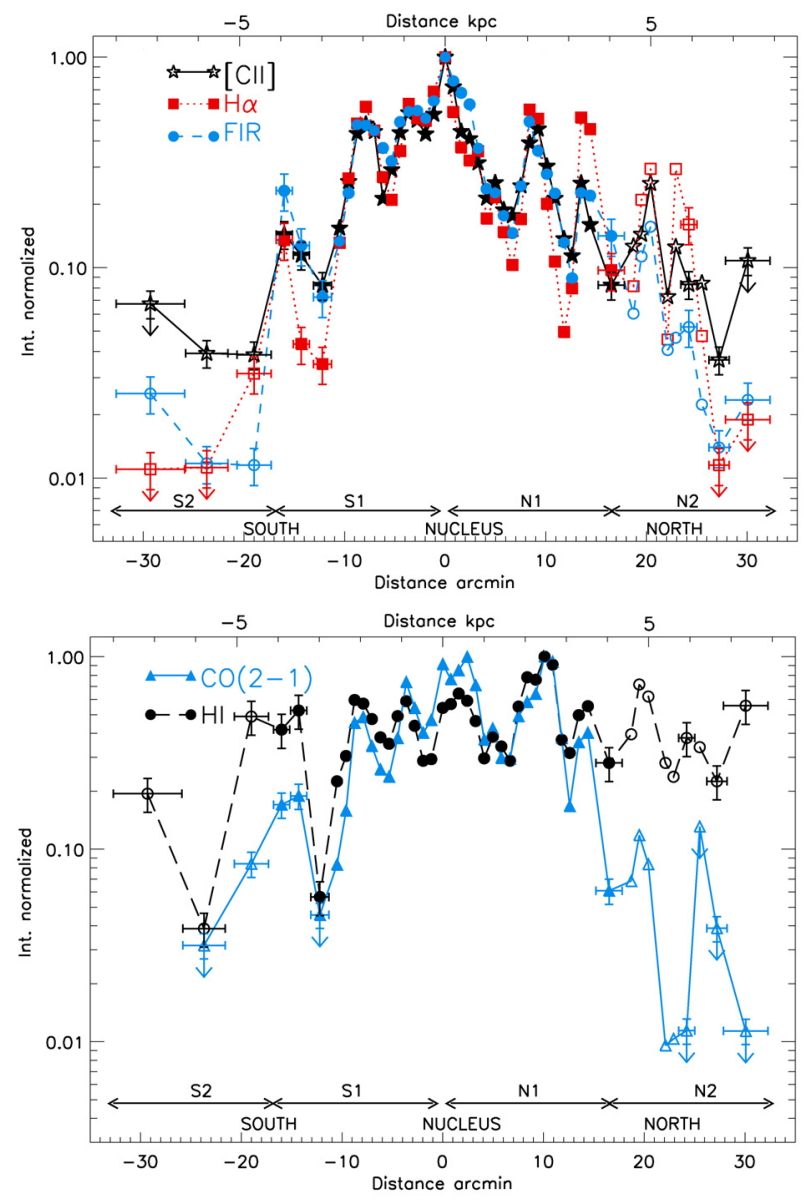

Fig. 2. Normalized integrated intensities of [C II], FIR, $\mathrm{H} \alpha$, CO 2-1, and $\mathrm{HI}$ along the major axis of M33. Closed symbols show data within $4.5 \mathrm{kpc}$ of the nucleus (N1, S1), and open symbols show observations in the outer galaxy $(\mathrm{N} 2, \mathrm{~S} 2)$. Horizontal errorbars show the region over which different [C II] spectra from neighboring positions were averaged.

\section{Results}

\subsection{Correlation among [CII], $\mathrm{FIR}, \mathrm{H} \alpha, \mathrm{CO}$, and $\mathrm{HI}$}

The emission of [C II], the FIR continuum, and $\mathrm{H} \alpha$ are all well correlated along the major axis, especially in the inner $10^{\prime}$ (Fig. 2). These tracers of star formation all peak at the nucleus and drop by more than one order of magnitude beyond $\sim 20^{\prime}$ radial distance. Closer inspection shows that the FIR continuum drops more steeply than [C II], which is discussed further below. In general, [C II], FIR, and $\mathrm{H} \alpha$ however all trace the spiral arms and the inter-arm regions. The close correlation is even more clearly seen in Fig. 3, where we plot the various tracers against [C II] emission. A close correlation of [C II] with other tracers of star formation is well-known for other sources and has also been seen in the ISO/LWS maps, which are a portion of the northern arm of M 31 by Rodriguez-Fernandez et al. (2006).

The atomic and molecular gas traced by $\mathrm{HI}$ and $\mathrm{CO}$ also drops with radial distance. However, the drop is much steeper for $\mathrm{CO}$ than for H I (Fig. 2). In the inner part of the galaxy that is within $10^{\prime}$ radial distance, the distribution of $\mathrm{CO}$ and $\mathrm{HI}$ is rather flat, which contrasts to that of the FIR continuum. The distribution of emission of both gas tracers is not symmetrical with respect to the nucleus. Instead, $\mathrm{CO}$ and $\mathrm{HI}$ peak near $10^{\prime}$ $(\sim 2.5 \mathrm{kpc})$ to the north and only show a secondary maximum at the nucleus. The absolute maximum corresponds to GMC 91 

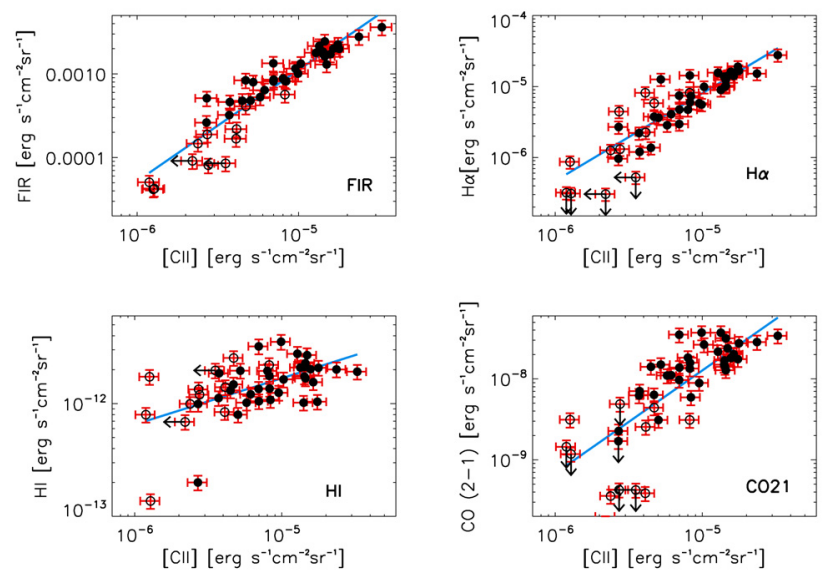

Fig. 3. Correlation of [C II] intensities of the FIR continuum, $\mathrm{H} \alpha$ emission, $\mathrm{CO}$, and $\mathrm{HI}$ in double-logarithmic plots. All intensities are given in units of erg s${ }^{-1} \mathrm{~cm}^{-2} \mathrm{sr}^{-1}$. Straight lines delineate the results of linear least-squares fits to the data. Closed and open symbols distinguish between positions in the inner (S1, N1) and outer (S2, N2) disk of M 33, respectively.

Table 2. Linear least-squares fits of the correlations of [C II], FIR, $\mathrm{H} \alpha$, $\mathrm{CO}$, and $\mathrm{HI}$ (cf. Fig. 3 ) in the form $\log \mathrm{FIR}=a+b \log [\mathrm{C}$ II $]$ with correlation coefficients $r$.

\begin{tabular}{ccccc}
\hline \hline & FIR-[C II] & $\mathrm{H} \alpha$-[C II] & $\mathrm{CO}(2-1)-[\mathrm{C} \mathrm{II}]$ & $\mathrm{H} \mathrm{I-[C} \mathrm{II]}$ \\
\hline$a$ & 3.71 & 1.08 & -1.52 & -9.74 \\
$b$ & 1.33 & 1.23 & 1.27 & 0.41 \\
$r$ & 0.90 & 0.79 & 0.59 & 0.30 \\
\hline
\end{tabular}

(Engargiola et al. 2003; Gratier et al. 2010; Buchbender et al. 2013) and to cloud 245 in Gratier et al. (2012).

Figure 3 shows correlations of [C II] emission, FIR, $\mathrm{H} \alpha, \mathrm{CO}$, and HI. Linear fits are weighted by the error along both axes, which we assume to be $15 \%$ for [C II] and $\mathrm{HI}$ and $20 \%$ for $\mathrm{CO}$ and the FIR continuum. The fits confirm that [C II] is strongly correlated with FIR and $\mathrm{H} \alpha$ using the linear correlation coefficients $(r) 0.90$ and 0.79 , respectively (Table 2 ). In contrast, the correlation with $\mathrm{CO}$ is much weaker where $r=0.59$, and very poor for H I where $r=0.30$. This result confirms that [C II] is a good tracer of the star formation rate at the ISO/LWS beam size scale. Points from the northern most region N2 deviate slightly from the fit both in the [C II]-FIR and the [C II]-H $\alpha$ plots which shows a worse correlation between [C II] and star-forming tracers in the northern outer galaxy.

\subsection{Radial variation of intensity ratios}

The radial distributions of the ratios $[\mathrm{C} \mathrm{II}] / \mathrm{CO} 1-0$, FIR/CO 1-0 and $\mathrm{HI} / \mathrm{CO} 1-0$ are shown in Fig. 4. We estimated CO 1-0 intensities from the $\mathrm{CO} 2-1$ line (cf. Appendix B). All three ratios show a minimum in the inner part of the galaxy and an increase towards the outer part. This observation reflects the steep drop of $\mathrm{CO}$ intensities already seen in the radial distribution of intensities (Fig. 2). The H I/CO ratio shows a minimum near the nucleus and rises steadily towards the outskirts over about two orders of magnitude. While this behavior is nearly symmetrical in the north and south of the galaxy, this symmetry is broken for the $[\mathrm{CII}] / \mathrm{CO}$ and FIR/CO ratios. The latter two ratios show a minimum at about $10^{\prime}$ to the north near GMC 91 where the $\mathrm{CO}$ emission peaks (Fig. 2). To the south of this minimum, the



Fig. 4. Normalized radial distributions of the $[\mathrm{C} \mathrm{II}] / \mathrm{CO} 1-0, \mathrm{FIR} / \mathrm{CO}$ $1-0$, and $\mathrm{HI} / \mathrm{CO} 1-0$ intensity ratios on the erg-scale.

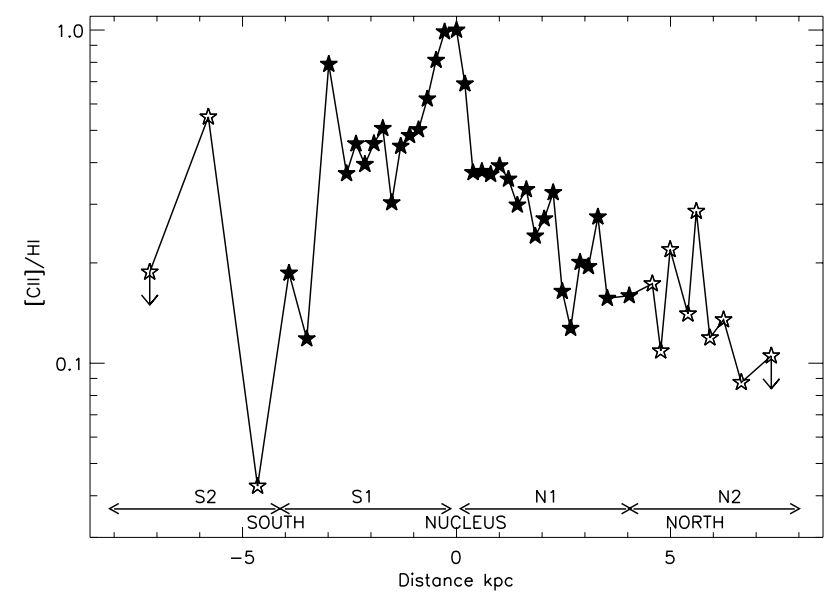

Fig. 5. Normalized radial distribution of the $[\mathrm{C} \mathrm{II}] / \mathrm{H} \mathrm{I}$ intensity ratio.

two ratios steadily increase. Towards the north, the two ratios also increases but with more scatter.

In general, the [C II] emission drops more steeply with galacto-centric radius than the H I emission. This drop is most clearly seen in the northern part of the strip (Fig. 5). In contrast, the southern outer disk shows strongly varying [C II]/H I ratios which owe to the strong variability of H I emission (cf. Fig. 2).

\section{3. $[\mathrm{CII}] /$ FIR ratio}

In the inner part of the galaxy $(\mathrm{N} 1, \mathrm{~S} 1)$, the $[\mathrm{CII}] / \mathrm{FIR}$ ratio stays constant at about $0.8 \% \pm 0.2 \%$ (Fig. 6) but rises significantly and steeply in the outskirts to values of $\sim 3 \%$. The rise of the $[\mathrm{C} \mathrm{II}] / \mathrm{FIR}$ ratio is caused by the steep drop of FIR emission relative to the [C II] (Fig. 2) which is also seen in a plot of $[\mathrm{C} \mathrm{II}] /$ FIR versus FIR (Fig. 7). The abrupt increase of the $[\mathrm{C} \mathrm{II}] / \mathrm{FIR}$ ratio occurs at about $4.5 \mathrm{kpc}$ radial distance in the north and in the south of the major axis. At this distance the morphology of the whole galaxy changes, as seen in optical images (Sharma et al. 2011). The change occurs just beyond the location of the prominent spiral arms. It also occurs in a region where the $\mathrm{HI} / \mathrm{CO}$ ratio rapidly increases (Fig.4). As the $\mathrm{O} / \mathrm{H}$ abundance gradient in M33 is shallow with a slope of only $\sim-0.035 \mathrm{dex} \mathrm{kpc}^{-1}$ and with no signs of a break 


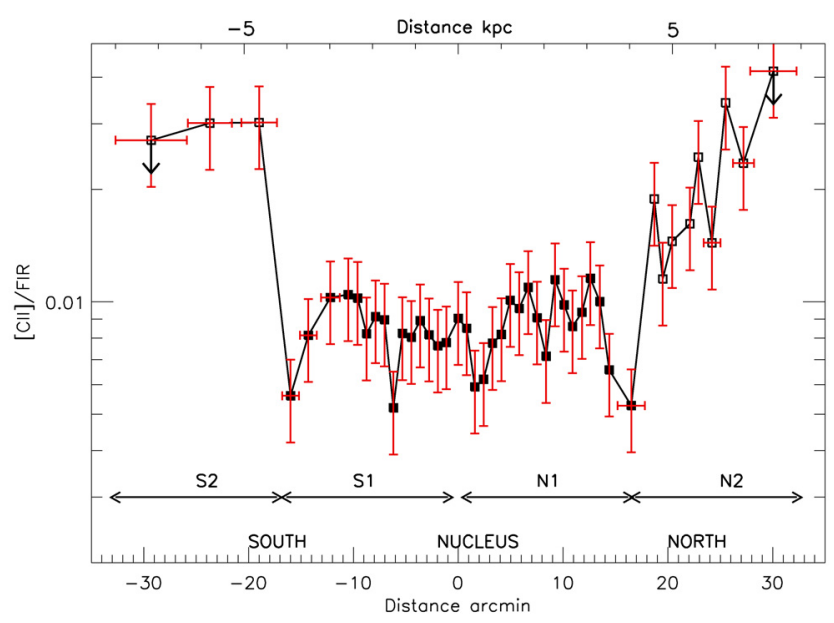

Fig. 6. Radial distribution of the $[\mathrm{C}$ II $] /$ FIR ratio along the major axis of M 33 .

(Magrini et al. 2010), the metallicity cannot be key for the sudden increase of the $[\mathrm{C}$ II]/FIR ratio.

Though only few studies of the radial variation of the $[\mathrm{C}$ II]/FIR ratio in galaxies exist, observations of M31, M 51, NGC 6946, and the Milky Way indicate that this ratio rises with galacto-centric distance. Rodriguez-Fernandez et al. (2006) present a discussion of ISO/LWS maps of a portion of the northern arm of M 31 at $12 \mathrm{kpc}$ distance from the nucleus. These data show a rather constant and high ratio of $2 \%$. In contast, the nucleus shows only $0.6 \%$. M 31 is at the same distance as M 33; the LWS observations of M31 sample the same linear scale as the present observations of M33. In a later section, we attempt to interpret the radial variation found along the major axis of M 33 using PDR models.

In M 33, the [C II]/FIR ratio drops with increasing FIR luminosity, $L_{\mathrm{FIR}}$. A power law fit to the entire data set results in $[\mathrm{C}$ II $] / \mathrm{FIR} \propto L_{\mathrm{FIR}}^{-0.34}(r=0.83)$. A fit to the data of the outer disk only results in a slightly steeper slope (Fig. 7). In this figure, we also compare the M 33 data with those of other galaxies. Relative to the normal galaxies observed by Malhotra et al. (2001) the M33 data are located in the region of low FIR luminosities $L_{\text {FIR }}=10^{5}-10^{7} L_{\odot}$ and high $[\mathrm{C} \mathrm{II}] /$ FIR ratios of between $0.6 \%$ and $3 \%$. The M 33 data lie in the same region as the data of the LMC, SMC and IC10.

The FIR luminosities of the low-metallicity systems are not directly comparable because their distances differ. However, the [C II] and FIR values of the LMC and SMC were taken from maps where the fluxes were averaged over individual sources from 35 to $70 \mathrm{pc}$ in size, while the data were taken at a range of 14 to $16 \mathrm{pc}$ linear resolutions (Israel et al. 1996; Israel \& Maloney 2011). The IC 10 observations have a resolution of $290 \mathrm{pc}$ (Madden et al. 1997), similar to that of the M 33 ISO/LWS data.

The M33 data in Fig. 7 lie in the high limit of [C II]/FIR ratios which are found in the sample of normal galaxies using ISO/LWS (Malhotra et al. 2001). The latter appears on the right side of the plot of $[\mathrm{C}$ II $] / \mathrm{FIR}=0.01 \%-0.7 \%$ and $L_{\mathrm{FIR}}=$ $10^{7}-10^{11} L_{\odot}$, which shows an average [C II]/FIR ratio of $0.32 \%$. The [C II]/FIR ratios in M 33 are 25-200 times higher than in Milky Way star-forming regions for a similar $L_{\mathrm{FIR}}$ range, while this factor decreases an order of magnitude (2.5-20) in Galactic GMCs.



Fig. 7. [C II]/FIR ratio as function of $L_{\mathrm{FIR}}$. The blue solid line shows a linear fit to the whole data set of M 33 while the green dotted line shows a linear fit to N2 and S2 points of the outer galaxy only. The black dashed-dotted horizontal line shows the lower [C II]/FIR value found in our data set of M33. The blue dotted horizontal line shows the average [C II]/FIR value in normal galaxies (Malhotra et al. 2001, M01). We also show data of Milky Way regions (Stacey et al. 1991, S91) and the lowmetallicity objects LMC, SMC, IC 10 (Israel et al. 1996, I96), (Israel \& Maloney 2011, I11), (Madden et al. 1997, M97).

\subsection{M33: A bridge between dwarf and normal galaxies?}

After comparing the $[\mathrm{C} \mathrm{II}] /$ FIR differences between M 33 and normal galaxies, we also compared $\mathrm{CO} / \mathrm{FIR}$ and $[\mathrm{C}$ II]/CO ratios found in M 33 with those of other sources (Fig. 8). In these plots, we find four mostly disjunct groups of ratios. There is a group formed by both low-metallicity objects and ratios of the outer galaxy of M 33 (N2, S2). The M 33 ratios of the inner galaxy $(\mathrm{N} 1, \mathrm{~S} 1)$ form another group, which connects low-metallicity objects with normal galaxies, and Milky Way GMCs (S91), which form the third group. Finally, PDRs in the Milky Way that are exposed to high FUV fields (MW SF regions, S91) form the fourth group. For normal galaxies, the $[\mathrm{C} \mathrm{II}] / \mathrm{CO} 1-0$ ratio increases with [C II]/FIR. For M 33 and other low-metallicity objects, the FIR/CO 1-0 ratios increase strongly with increasing [C II $] / \mathrm{CO} 1-0$. This correlation is also seen for normal galaxies, though with increased scatter. The data points of the inner disk of M 33 lie between the data of the normal galaxies at low [C II $] / \mathrm{CO}$ ratios and the data of the other low-metallicity systems, which show high $[\mathrm{C} \mathrm{II}] / \mathrm{CO}$ ratios.

On average, the inner parts of M 33 (N1, S1) exhibit lower [C II]/CO and FIR/CO ratios than the other low-metallicity objects. This finding suggests that $\mathrm{CO}$ is less photo-dissociated in the inner disk of M 33 than in the outer regions (S2, N2) and than in the other low-metallicity objects. In the next paragraphs we will compare the observed ratios with the predictions of PDR models.

\section{Discussion}

\subsection{Diagnostic diagram of [CII]/FIR vs. CO/FIR}

In Fig. 9, we plot luminosities of [C II] versus $\mathrm{CO}$, which are normalized with FIR luminosities. To this diagnostic diagram, we added observations of other galaxies, the corresponding lines of constant FUV-fields, and local volume densities of standard K99 PDR models which use a cloud optical extinction of $A_{\mathrm{V}}=10 \mathrm{mag}$ and solar metallicities. For completeness, we show 

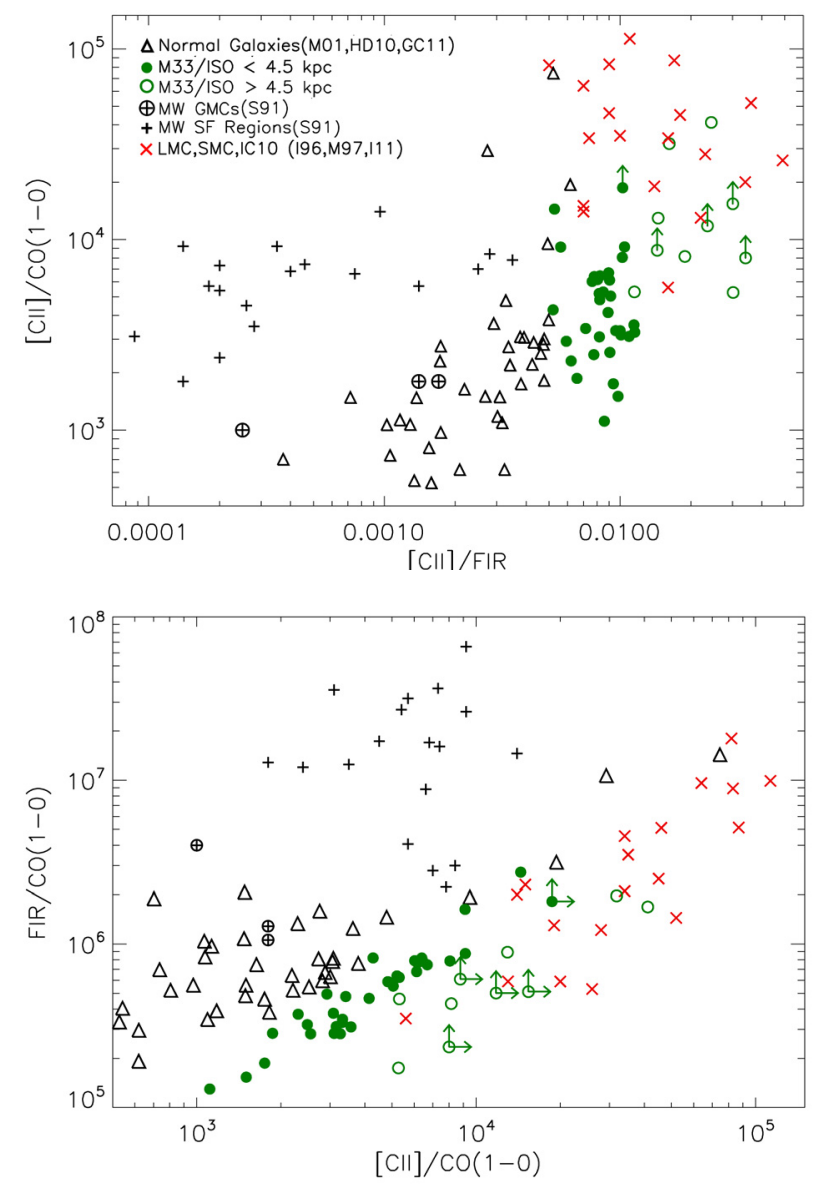

Fig. 8. Diagnostic plots of the ratios of [C II], $\mathrm{CO}$ and the FIR continuum for M33 and for four other types of objects, low-metallicity systems, normal galaxies, Milky Way star-forming regions, and GMCs. Upper panel: [C II]/CO 1-0 vs. [C II]/FIR. Bottom panel: FIR/CO 1-0 vs. [C II]/CO 1-0. All panels are shown on a logarithmic scale. Symbols used have the same meaning than those used in Fig. 7.

Table 3. Range of densities and FUV fields consistent with the standard K99 PDR model for sources that are shown in the diagnostic plot of [C II]/FIR vs. CO/FIR (Fig. 9).

\begin{tabular}{lccc}
\hline \hline Objects & {$[\mathrm{C} \mathrm{II}] /(\mathrm{CO} 1-0)$} & $n\left[\mathrm{~cm}^{-3}\right]$ & $G_{0}$ \\
\hline M33 (N1-S1) & $1000-8000$ & $<10^{4}$ & $<10^{3}$ \\
M 33 (N2-S2) & $5000-4.110^{4}$ & - & - \\
MW-SF & $1000-5000$ & $10^{2}-10^{6}$ & $10^{3}->10^{4}$ \\
MW-GMCs & $500-1000$ & $10^{5}-10^{6}$ & $500-\gtrsim 10^{3}$ \\
Normal galaxies & $500-4000$ & $10^{3}-10^{6}$ & $10^{2}-10^{3}$ \\
ULIRGs & $500-4000$ & $10^{4}-10^{6}$ & $10^{3}-10^{4}$ \\
Starburst nuclei & $\sim 4100$ & $10^{3}-10^{4}$ & $500-\gtrsim 10^{3}$ \\
Non-starburst nuclei & $500-4000$ & $10^{5}-10^{5}$ & $10^{2}-10^{3}$ \\
LMC, SMC, IC 10 & $8000-10^{5}$ & - & - \\
\hline
\end{tabular}

Notes. For the outer regions of M33 and for the LMC, SMC, and IC 10, the standard model fails.

[C II] vs. CO normalized with the total-infrared (TIR) luminosities in Appendix D.

\subsubsection{Observations}

M33. The $[\mathrm{CII}] /$ FIR ratios, which are observed on scales of $280 \mathrm{pc}$ in $\mathrm{M} 33$, stay rather constant at $\sim 0.8 \%$ in the inner parts of $\mathrm{M} 33$, and rise to $3 \%$ in the outer regions, as already presented in the previous sections. The $[\mathrm{C} \mathrm{II}] / \mathrm{CO} 1-0$ ratios vary between 1000 and 41200 , while the CO $1-0 /$ FIR ratios vary between $4 \times 10^{-7}$ and $8 \times 10^{-6}$.

On scales of $50 \mathrm{pc}\left(12^{\prime \prime}\right)$, the [C II]/FIR ratio varies between $0.01 \%$ and $3 \%$ over the $2^{\prime} \times 2^{\prime}$ map of the BCLMP $302 \mathrm{H}$ II region, as seen in Mookerjea et al. $(2011, \mathrm{M} 11)^{3}$. The bulk of [C II]/FIR ratios lie in the range of $\sim 0.7 \%-1 \%$. The CO $1-0 /$ FIR ratios lie between $4 \times 10^{-7}$ and $8 \times 10^{-6}$. Interestingly, the PACS observations of the $2^{\prime} \times 2^{\prime}$ BCLMP 302 region, which lies at a galacto-centric distance of $2.1 \mathrm{kpc}$, cover the same range of $[\mathrm{C}$ II $] /$ FIR and $\mathrm{CO} 1-0 / \mathrm{FIR}$ ratios, which are found with ISO/LWS along the entire major axis of M 33 up to $8 \mathrm{kpc}$.

Other galaxies. The $[\mathrm{C} \mathrm{II}] / \mathrm{CO}$ ratios found in the inner parts of M 33 lie in the same range of values, which are 1 found in the bulk of the normal galaxies and ULIRGs shown here. The outer regions of M 33 show higher values, similar to those found in other low-metallicity systems.

The ISO/LWS [C II]/FIR ratios of M 33 are higher than in normal galaxies, which only exhibit [C II]/FIR ratios of up to $0.4 \%$. The low-metallicity galaxies LMC, SMC, and IC 10 show high [C II]/FIR ratios, which are comparable to those found in M 33, as already discussed above. Local ULIRGs show CO/FIR ratios of less than $10^{-6}$, while normal galaxies show higher ratios of up to $5 \times 10^{-6}$. M33 shows slightly higher peak ratios of up to $8 \times 10^{-6}$.

\subsubsection{PDR model results}

Observations consistent with the standard model. Most of the [C II]/FIR and CO/FIR ratios observed in the inner disk of M 33 lie in the parameter space of local densities and FUV fields spanned by the standard PDR of K99 (solar metallicity $Z=1.0$, $A_{V}=10 \mathrm{mag}$, Fig. 9). These ratios indicate densities of less than $\sim 10^{4} \mathrm{~cm}^{-3}$ and FUV fields of less than $G_{0} \sim 10^{3}$ in units of the local interstellar value. Some of the ratios observed in the inner galaxy and all ratios observed in the outer disk are, however, not consistent with this standard model, as discussed below.

In the literature, the observed $\mathrm{CO}$ intensities have sometimes been multiplied by a factor of two for the comparison with the predictions of the Kaufman PDR model. Hailey-Dunsheath et al. (2010) argue that CO 1-0 is optically thick, stemming only from the front-side of FUV illuminated clouds, while the FIR emission is in general optically thin, stemming from the front and the backside of clouds. We do not apply any factor; instead we use the observed values and argue that the optical depth of the galactic CO emission on scales of 280 pc will be reduced because of the velocity dispersion by turbulence and the large-scale gradients and that an ad-hoc factor of two does not seem appropriate.

In the framework of the standard PDR model, normal galaxies, especially the ULIRGs, tend to be consistent with higher densities that are up to a few times of $10^{6} \mathrm{~cm}^{-3}$ than that are typically found in M33. The latter are also consistent with higher FUV fields of up to $\sim 10^{4}$. Table 3 summarizes the range of values returned by the standard K99 PDR model for the whole data sample shown in Fig. 9. Normal galaxy and starburst nuclei points with extreme high [C II]/CO values were ignored here.

3 To convert the TIR values used by M11 into FIR, we used the
FIR/TIR ratios derived from the greybody fits (Sect. 2.2, Table B.1).
Two ISO/LWS positions, N49 and N50, lie in the region mapped by M11. We took the average FIR/TIR value for the conversion. 
C. Kramer et al.: Gas and dust cooling along the major axis of M33 (HerM33es)

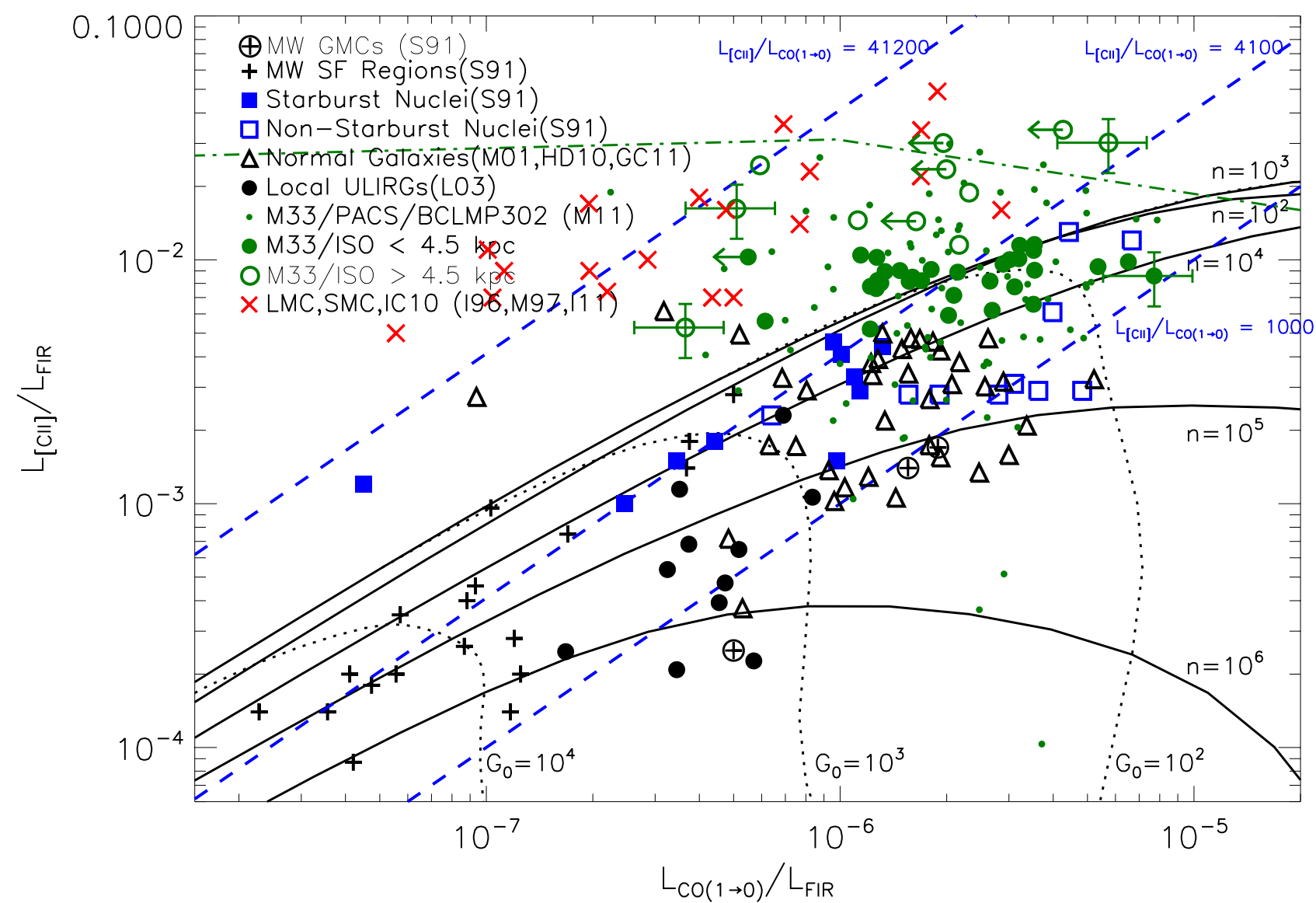

Fig. 9. [C II] versus CO, which are normalized with the FIR continuum. Big green filled circles show ISO/LWS data of the inner S1, N1 regions of M33, while open circles show data of the outer S2, N2 regions. Small green circles show PACS observations of the BCLMP 302 H II region in M 33 (M11). In addition we show data from Milky Way GMCs, from star-forming regions, and from other galaxies, which are compiled from Stacey et al. (1991, S91), Hailey-Dunsheath et al. (2010, HD10), Graciá-Carpio et al. (2011, GC11), Israel et al. (1996, I96), Israel \& Maloney (2011, I11), Madden et al. (1997, M97), Malhotra et al. (2001, M01), and Luhman et al. (2003, L03). The lowest [C II]/CO ratio observed with ISO/LWS in M 33 is 1000 (lower blue dashed line), while the highest ratio is 41200 (upper blue dashed line). Black solid and dotted lines indicate lines of constant density $n$ and FUV field $G_{0}$, respectively, from the standard K99 PDR model with $A_{\mathrm{V}}=10$ mag and solar metallicity $Z=1$. The dashed-dotted green line shows a K99 PDR model result for $A_{\mathrm{V}}=1 \mathrm{mag}, Z=1$, and $n=10^{3} \mathrm{~cm}^{-3}$. The knee of this curve is for $G_{0}=10^{0.5}$ (cf. Fig. 18 in K99).

Observations inconsistent with the standard model. All ISO/LWS [C II]/FIR and CO/FIR ratios of the outer disk of M 33 are inconsistent with the standard PDR model for any density and FUV field. In general, [C II]/FIR ratios above $2 \%$ cannot be reproduced by the standard model for $\mathrm{CO} / \mathrm{FIR}$ ratios which are less than $10^{-5}$. For lower CO/FIR ratios, the upper limit of $[\mathrm{C}$ II $] /$ FIR, which can be modeled, drops. For instance, the upper limit of [C II]/FIR lies near $0.5 \%$ at CO/FIR $\sim 10^{-6}$.

The scatter of $[\mathrm{C}$ II]/FIR and CO/FIR data seen in M33 on the large scales sampled by the ISO/LWS beam is similar to the scatter seen on small scales of $50 \mathrm{pc}$ in the small subregion BCLMP 302. However, variations of metallicities are not expected on such small scales. The observed scatter of [C II]/FIR and $\mathrm{CO} / \mathrm{FIR}$ ratio must therefore reflect the variation of other properties of the emitting gas and dust.

As shown in Figs. 9 and 10, K99 PDR models of low optical extinctions reproduce the high [C II]/FIR ratios observed predominantly in the outer disk of M33. Assuming a total column density that corresponds to an $A_{\mathrm{V}}$ of only 1 mag and to solar metallicity, the ratios observed in the outer disk are consistent with $n \sim 10^{3} \mathrm{~cm}^{-3}$ and $G_{0} \sim 1$, while the ratios observed in the inner disk are consistent with somewhat higher densities and
FUV fields of $n \sim 10^{4} \mathrm{~cm}^{-3}$ and $G_{0} \sim 10$ (Fig. 10). The ratio of $G_{0} / n$ is about $10^{-3} \mathrm{~cm}^{3}$ in both cases.

Subsolar metallicities are not needed to explain these data sets, because the curves for the models with subsolar metallicity $Z=0.1$ are similar to the curves for $Z=1.0$. This finding also shows that $[\mathrm{C} \mathrm{II}]$ is relatively insensitive to changes in the metallicity. When [C II] dominates gascooling, the models indicate that the gas temperature of the PDR surface layer, which emits [C II] adjusts in a way that the emergent [C II] flux equals the FUV flux times the gas heating efficiency (Kaufman et al. 2006).

Because the observed metallicity gradient of M 33 is shallow (Magrini et al. 2010), we do not suspect that the average optical extinction of clouds changes abruptly from about 10 mag to about 1 mag between the inner and outer disk of M33. We suggest, instead, that the overall low-metallicity environment of M 33 is composed of clouds that have generally low optical extinctions of one or a few magnitudes.

In this scenario, the rather abrupt increase of heating efficiency beyond about $4.5 \mathrm{kpc}$ radial distance is caused by a drop of average local densities of the molecular gas and a decrease of FUV field strengths. This drop of the FUV along the major axis of M33 is already indicated by the radially averaged extinction 




Fig. 10. [C II] versus CO, which are normalized with the FIR continuum (Fig. 18 of K99). Black solid and dotted lines indicate lines of constant density for metallicities of $Z=1.0$ and $Z=0.1$, respectivey, from the K99 PDR model using $A_{\mathrm{V}}=1 \mathrm{mag}$. Big green filled circles show ISO/LWS data of the inner S1, N1 regions of M33, while open circles show data of the outer $\mathrm{S} 2, \mathrm{~N} 2$ regions.

corrected FUV fluxes (cf. Fig. 3 in Verley et al. 2009). Verley et al. (2007) did IR photometry of 515 compact sources. The best models to reproduce the extinction seen in these $\mathrm{H}$ II regions are the ones with $A_{\mathrm{V}}<10 \mathrm{mag}$.

The change of density from $10^{4}$ in the inner disk to $10^{3}$ in the outer disk would imply that PDRs in the outer disk are typically about a factor of 10 larger in size than in the inner disk:

$\frac{r_{\text {outer }}}{r_{\text {inner }}}=\frac{N\left(\mathrm{H}_{2}\right)_{\text {outer }} / n\left(\mathrm{H}_{2}\right)_{\text {outer }}}{N\left(\mathrm{H}_{2}\right)_{\text {inner }} / n\left(\mathrm{H}_{2}\right)_{\text {inner }}} \sim 10$.

We estimated the FUV field in Habing units (Habing 1968) directly from the FIR continuum. We used $G_{0}=4 \pi I_{\mathrm{FIR}} / 1.6 \times 10^{-3}$ in erg s${ }^{-1} \mathrm{~cm}^{-2}$ (Mookerjea et al. 2011; Kaufman et al. 1999) and obtained $G_{0}=28$ for the nucleus and $G_{0}=1.3$ for the N66 position in the outer disk of M 33. The low FUV field found in the outer disk is consistent with the radiation field predicted by the K99 PDR model from the [C II]/FIR and CO/FIR ratios for low extinctions.

For a fixed CO/FIR ratio, a given density, and a given metallicity, the modelled [C II]/FIR decreases with increasing optical extinction (Figs. 16-18 in Kaufman et al. 1999). For example, $\log ([\mathrm{C}$ II $] /$ FIR $)$ decreases by a factor $\sim 5$ from -1.5 at 1 mag to -2.2 at $10 \mathrm{mag}$ at $\log (\mathrm{CO} / \mathrm{FIR})=6$, In clouds of low optical extinctions, FUV photons penetrate deeper into the cloud. For spherical clouds, the smaller $\mathrm{CO}$ core is surrounded by a larger [C II] emitting region, which leads to enhanced [C II]/CO ratios (cf. Bolatto et al. 1999; Röllig et al. 2006). The observed high $[\mathrm{C}$ II $] /$ FIR and $[\mathrm{C} \mathrm{II}] / \mathrm{CO}$ ratios are best explained by clouds of low columns (optical extinctions) which lead to an increase of the $[\mathrm{C} \mathrm{II}]$ layer relative to the total gas traced by $\mathrm{CO}$. Such clouds also lead to an increase of the [C II] emission relative to the total dust traced by the FIR continuum. We propose that reduced volume densities and the geometrical dilution of the FUV field,

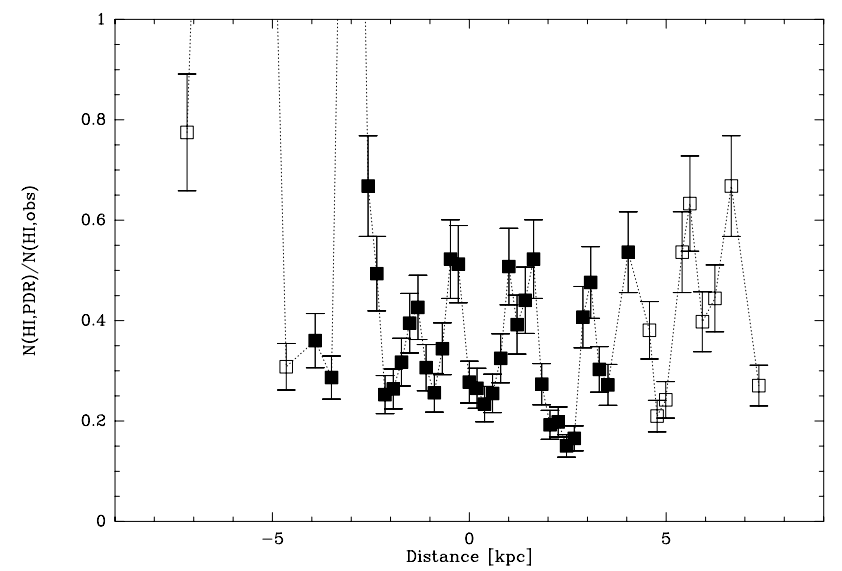

Fig. 11. Fraction of Hi column density, which stem from PDRs. Errorbars only show the $15 \%$ observational error of $\mathrm{HI}$ intensities. Two positions in the south, where $N(\mathrm{H} \mathrm{I}, \mathrm{PDR})>N(\mathrm{H} \mathrm{I}, \mathrm{obs})$, are not shown.

when photons can enter more deeply, are of secondary importance only.

The low optical extinctions are consistent with a reduced dustto-gas ratio in the low-metallicity environment of M33. As discussed by Israel et al. (1996) and Israel \& Maloney (2011) using data of the $\mathrm{LMC}$, the ratio of total gas column density over optical extinction, $N_{\mathrm{H}} / A_{\mathrm{V}}$, increases in low-metallicity environments.

\subsection{HI emission from PDRs}

In the following, we assume that all observed $\mathrm{H}$ I emission stems from the cold neutral and atomic medium (CNM) and from PDRs. Before discussing a possible contribution of [C II] emission from the CNM, we first need to study the $\mathrm{H}$ I fraction, which stem from PDRs. At the surface of PDRs, molecular hydrogen is photodissociated and a layer of atomic hydrogen is formed. The column from $\mathrm{H} \mathrm{I}$ is a function of impinging FUV field over the volume density of the molecular gas, $G_{0} / n$, as shown e.g. by Sternberg (1988). Stacey et al. (1991) studied the contribution of H I emission from PDRs to the total hydrogen column in a sample of galaxies. With the typical densities and FUV fields derived above for M 33, we find a typical $G_{0} / n$ ratio of $10^{-3} \mathrm{~cm}^{3}$ in the inner and outer disk of M33. Next, we use Eq. (3) of Heiner et al. (2011):

$N(\mathrm{HI}, \mathrm{PDR})=\frac{7.8 \times 10^{20}}{D} \ln \left(1+\frac{106 G_{0}}{n} D^{-0.5}\right) \mathrm{cm}^{-2}$

where the dust-to-gas ratio $D$ is normalized to the solar neighborhoud value of $(12+\log (\mathrm{O} / \mathrm{H}))-8.69$ (Eq. (2) of Heiner et al. 2011) and a constant oxygen abundance of $12+\log (\mathrm{O} / \mathrm{H})=8.27$ (see references in Buchbender et al. 2013). This calculation leads to a constant Hi column density, which stem from PDRs of $3.25 \times 10^{20} \mathrm{~cm}^{-2}$.

In Fig. 11, we compare the H I column density from PDRs to the observed beam-averaged H I column density, assuming that the PDRs fill the beam. We did not attempt detailed PDR modeling to derive the beamfilling factor (e.g. by comparing the observed FIR continuum with the best fitting FUV field from models). Stacey et al. (1991) derived a typical beamfilling factor of 0.3 for the sample of galaxies they studied. In M33, the estimated fraction of H I column densities, which stem from PDRs stays between $15 \%$ and $70 \%$ for all data between $2 \mathrm{kpc}$ radial 
distance in the south and $7.3 \mathrm{kpc}$ in the north. Two positions further south exhibit very low H I columns, which are lower than the estimated H I column from PDRs; their $N(\mathrm{H} \mathrm{I}, \mathrm{PDR}) / N(\mathrm{H} \mathrm{I}, \mathrm{obs})$ ratios rise above 1 to values of up to 4 , which indicates that the underlying assumptions of $G_{0}, n$, and the beam filling factor are not valid for these regions.

\section{3. [CII] from the atomic medium}

We investigate here which fraction of the observed [C II] emission could stem from the atomic cold neutral medium (CNM) in M33. As described in Sect.3.1, the emission of H I drops only weakly towards the outskirts, while the $\mathrm{CO}$ emission drops steeply (Fig. 2). In the southern part of the strip, the $\mathrm{HI} / \mathrm{CO}$ rises continuously from a minimum in the center towards the outskirts at about a radial distance of $5 \mathrm{kpc}$ (Fig. 4). On the other hand, the $\mathrm{HI} / \mathrm{CO}$ in the north shows an abrupt increase at about a radial distance of $4.5 \mathrm{kpc}$, which is the same distance where the $[\mathrm{C} \mathrm{II}] /$ FIR ratio rises to values of $\sim 3 \%$ (Fig. 6 ). The relative contribution of the atomic gas that traces the CNM to the total gas emission $(\mathrm{CO}+\mathrm{HI})$ rises with galacto-centric distance. The $\mathrm{HI} /[\mathrm{C} \mathrm{II}]$ intensity ratio also rises strongly in the northern part of the strip over almost an order of magnitude (Fig. 5). This rise is because the $[\mathrm{C} \mathrm{II}]$ emission drops more steeply with the radius than the $\mathrm{HI}$. In the north, the observed [C II] emission has dropped by a factor of 27 relative to its value at the nucleus, while H I has dropped only by $40 \%$. Any contribution of the $\mathrm{CNM}$ to the $[\mathrm{C}$ II] emission relative to the contribution from PDRs should be most prominent in the outskirts.

We note that the correlation between $\mathrm{HI}$ and [C II] emission is rather weak throughout the strip and also in the outskirts (cf. Fig. 3). Mookerjea et al. (2011) and Braine et al. (2012) studied [C II] HIFI spectra in two regions of $\sim 1^{\prime}-2^{\prime}$ along the major axis of M33 at radial distances of 2.1 and $3.3 \mathrm{kpc}$. In both sources, the $\mathrm{HI}$ lines are systematically shifted relative to the [C II] lines by about $5 \mathrm{kms}^{-1}$. In addition, $\mathrm{H}$ I linewidths are about $30 \%$ broader than those of [C II]. These findings indicate that the $[\mathrm{C} \mathrm{II}]$ emission, in at least these two regions of the inner galaxy, does not trace only the atomic medium.

To quantify a possible contribution of the cold neutral medium (CNM) to the [C II] emission, we use the $\mathrm{H}$ I line intensity, which is corrected for the contribution from PDRs, to derive the intensity of the [C II] line (cf. Crawford et al. 1985; Madden et al. 1993, 1997; Langer et al. 2010). Here, we assume optically thin emission of [C II] and $\mathrm{HI}$, and beamfilling factors of unity. We estimate the $[\mathrm{C} \mathrm{II}]$ intensities, which stem from the remaining fraction of the atomic gas via

$$
\begin{aligned}
I^{\prime}(\mathrm{HI})= & 3.35 \times 10^{14} I(\mathrm{HI}) \\
N(\mathrm{HI})= & 1.82 \times 10^{18} I^{\prime}(\mathrm{HI}) \\
N(\mathrm{HI}, \mathrm{CNM})= & N(\mathrm{HI})-N(\mathrm{HI}, \mathrm{PDR}) \\
N\left(\mathrm{C}^{+}, \mathrm{CNM}\right)= & X_{\mathrm{C}^{+}} N(\mathrm{HI}, \mathrm{CNM}) \\
I\left(\mathrm{C}^{+}, \mathrm{CNM}\right)= & 2.35 \times 10^{-21} N\left(\mathrm{C}^{+}\right) \\
& \times\left(\frac{2 \exp (-\Delta E / T)}{1+2 \exp (-\Delta E / T)+\left(n_{\mathrm{cr}} / n\right)}\right),
\end{aligned}
$$

where intensities $I$ are in units of $\mathrm{erg} \mathrm{s}^{-1} \mathrm{~cm}^{-2} \mathrm{sr}^{-1}, I^{\prime}$ in $\mathrm{K} \mathrm{km} \mathrm{s}^{-1}$, and column densities in $\mathrm{cm}^{-2}$. The energy of the [C II ${ }^{2} P_{3 / 2}$ state above ground is $\Delta E=h v / k=91.3 \mathrm{~K}$, and the critical density for collisions with hydrogen atoms and/or molecules is $n_{\mathrm{cr}}=3 \times 10^{3} \mathrm{~cm}^{-3}$ (cf. discussion and references in Langer et al. 2010). The fractional abundance $X\left(\mathrm{C}^{+}\right)$ of $\mathrm{C}^{+}$in the gas with respect to $\mathrm{HI}$ lies in the range of 1.4

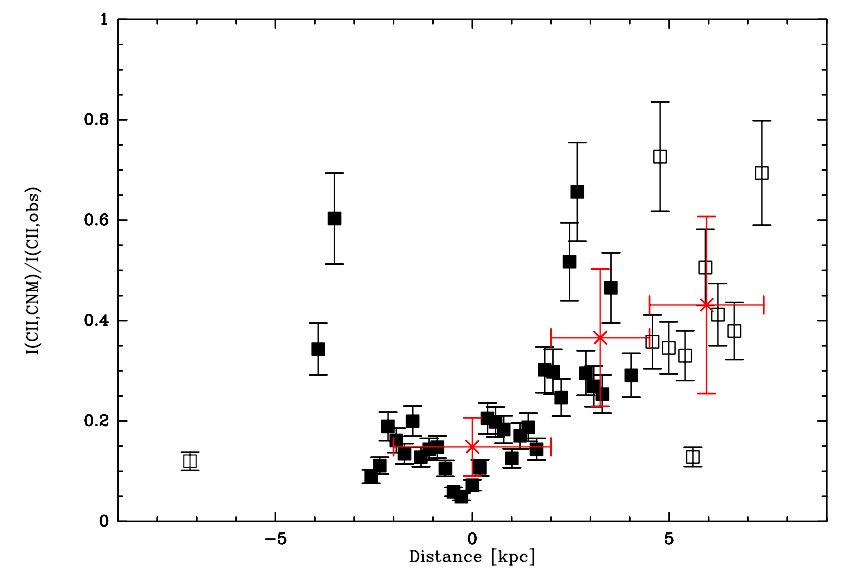

Fig. 12. Fraction of the observed $[\mathrm{C} \mathrm{II}]$ emission stemming from the cold neutral medium for $T=80 \mathrm{~K}$ and $n=100 \mathrm{~cm}^{-3}$, after correction of the observed H I for the contribution from PDRs. Squares mark the individual data along the major axis. Their errorbars only include the $15 \%$ observational error of the $\mathrm{HI}$ intensities. Crosses in red mark the fractions averaged over the inner $2 \mathrm{kpc}$, the northern 2 to $4.5 \mathrm{kpc}$, and the outer, northern disk between 4.5 and $7.4 \mathrm{kpc}$. Corresponding errorbars show the standard deviation of the individual data and the radius interval over which the values were binned.

to $1.8 \times 10^{-4}$ in the local ISM of the Milky Way (Sofia et al. 1997). For the average low-metallicity environment of M33, we assume $X\left(\mathrm{C}^{+}\right)=0.6 \times 10^{-4}$ (Magrini et al. 2010; Henry et al. 2000). In addition, we assume diffuse atomic clouds of $n=100 \mathrm{~cm}^{-3}$ and $T=80 \mathrm{~K}$, which corresponds to a pressure of about $8000 \mathrm{~K} \mathrm{~cm}^{-3}$ and is typical for the diffuse atomic medium in the Milky Way (e.g. Wolfire et al. 1995; Dickey et al. 2000). Higher temperatures and densities would increase the $[\mathrm{CII}]$ intensities but quickly become inconsistent with the generally accepted values for the pressure of the Galactic atomic ISM (cf. discussion in Sect. 4.2.1. of Madden et al. 1993).

Figure 12 shows the estimated fraction of the observed [C II] intensity, which stems from the CNM. As expected from the observed ratio of $\mathrm{HI}$ over [C II] intensities, the fraction is lowest in the nucleus and generally rises towards the outskirts. Towards the southern half, the CNM fraction shows strong variability, reflecting the variations of $\mathrm{H}$ I emission on small scales (cf. Fig. 2). However, the fraction rises towards the north generally from $20 \% \pm 6 \%$ in the inner $2 \mathrm{kpc}$, to $37 \% \pm 14 \%$ for radii between 2 and $4.5 \mathrm{kpc}$, to $43 \% \pm 18 \%$ for radii between 4.5 and $7.4 \mathrm{kpc}$.

The rise of [C II]/FIR by a factor of more than three may therefore be partially explained by a rising contribution of the CNM to the $[\mathrm{C}$ II $]$ emission, while the $[\mathrm{C}$ II $] /$ FIR ratio of PDRs rises only moderately with only somewhat lowered values of $A_{V}, n$, and $G_{0}$ relative to the PDR models for the inner galaxy of $A_{V} \sim 10 \mathrm{mag}$, $n \sim 10^{4} \mathrm{~cm}^{-3}$, and $G_{0} \sim 10$. A better knowledge of the temperature and density of the CNM is needed to derive more quantitative conclusions. We conclude that PDRs dominate in the inner parts of M33, while both the CNM and PDRs can explain the [C II] emission observed in the outskirts.

\subsection{The importance of the Oı $63 \mu \mathrm{m}$ line}

A more complete study of the major gas cooling lines of the ISM would need to include the [O I] $63 \mu \mathrm{m}$ line as a tracer of high densities in PDRs. Higdon et al. (2003) detected this line at scales of $280 \mathrm{pc}$ in the nucleus and toward five $\mathrm{H}$ II regions in M 33, where they found [C II $] /[\mathrm{O} \mathrm{I}]$ ratios between 1.6 and 2.2. 
Toward the H II region BCLMP 302 and on scales of $50 \mathrm{pc}$, the ratio varies betwen 2.5 and 10 (Mookerjea et al. 2011). Further Herschel/PACS and HIFI observations of several regions along the major axis of M 33 have been conducted in the framework of the HerM33es project to obtain a more complete dataset, which will allow more accurate estimates of the photoelectric heating efficiency, its variation on small scales, and the determination of gas properties $\left(A_{V}, n, T\right)$ using PDR models.

\section{Summary and conclusions}

In this paper, we have studied ISO/LWS [C II] emission along the major axis of M 33 at scales of 280 pc. Stacking of few adjacent positions allowed detection of [C II] between galacto-centric distances of $\sim 6 \mathrm{kpc}$ in the south to $\sim 6.7 \mathrm{kpc}$ in the north. The radial distributions of the $[\mathrm{C} \mathrm{II}] / \mathrm{CO}$ and $\mathrm{H} \mathrm{I} / \mathrm{CO}$ ratios show an increase from the inner regions to the outer regions. While the $\mathrm{HI} / \mathrm{CO}$ ratio has a minimum near the nucleus, the $[\mathrm{C} \mathrm{II}] / \mathrm{CO}$ ratio shows a minimum at about $10^{\prime}$ to the north, which corresponds to the global maximum of $\mathrm{CO}$ emission along the major axis.

The radial distribution of [C II] shows a strong correlation with star-formation tracers, the FIR continuum, and $\mathrm{H} \alpha$ emission, which closely follows the spiral arm structure. The correlation becomes weaker in the outskirts of M33 beyond a galactocentric distance of $4.5 \mathrm{kpc}$.

The $[\mathrm{C}$ II $] /$ FIR ratio remains constant at $\sim 0.8 \%$ in the central $4.5 \mathrm{kpc}$ radius and then increases rapidly to values of up to $3 \%$. The [C II]/FIR ratio is a strong function of the FIR luminosity. The highest $[\mathrm{C}$ II]/FIR ratios are found for the lowest FIR luminosities. Other low-metallicity systems (LMC, SMC, IC 10) show similar [C II]/FIR ratios to M33, which are higher than those found in the Milky Way and in normal galaxies.

The variation of $[\mathrm{CII}] / \mathrm{FIR}$ and CO/FIR ratios found in a small $2^{\prime} \times 2^{\prime} \mathrm{HII}$ region of M33 on small scales of $50 \mathrm{pc}$ (Mookerjea et al. 2011) is very similar to the variation found with the ISO/LWS observations. This similarity is another indication that metallicity variations play a minor role in determining the $[\mathrm{C} \mathrm{II}] /$ FIR ratio.

Diagnostic plots of $[\mathrm{CII}] / \mathrm{FIR}$ vs. CO/FIR or FIR/CO vs. $[\mathrm{C}$ II $] / \mathrm{CO}$ show smooth transitions from normal galaxies to the positions in the inner $4.5 \mathrm{kpc}$ radius of M33 and to the outer parts of M 33 together with the other low-metallicity systems. Observations of the inner disk of M 33 serve as a bridge between normal galaxies exhibiting low [C II]/FIR and low [C II]/CO ratios to the low-metallicity systems, which show high [C II]/FIR and high $[\mathrm{C} \mathrm{II}] / \mathrm{CO}$ ratios.

The relative lack of dust shielding in the low-metallicity systems enhances $\mathrm{CO}$ photo-dissociation, hence, increases the [C II]/CO ratios. The standard K99 PDR models of 10 mag optical extinction fail to reproduce the high end of the observed $[\mathrm{C} \mathrm{II}] /$ FIR and [C II]/CO ratios. However, models of low optical extinction expected in the low-metallicity environment of M33 do reproduce the observed ratios for a constant metallicity. In the framework of models where $A_{V}=1 \mathrm{mag}$, the variation of observed $[\mathrm{C} \mathrm{II}] /$ FIR ratios is caused by variations of the local densities that drop from about $10^{4} \mathrm{~cm}^{-3}$ in the inner disk to $10^{3} \mathrm{~cm}^{-3}$ in the outer parts. In addition, FUV field strengths reach to about 10 times the average interstellar radiation field in the inner disk, while dropping to only a few in the outskirts. High FUV fields tend to lower the photoelectric heating efficiency as grains and PAHs become positively charged (Okada et al. 2013). In contrast, the high $[\mathrm{C} \mathrm{II}] /$ FIR ratios observed in low-metallicity galaxies can be caused by geometric dilution of low FUV fields in clouds of normal densities where carbon stays ionized.

In addition to PDRs, the atomic CNM traced by $\mathrm{H}$ I may contribute to the observed [C II] emission. Therefore, the above scenario may need to be revised. We conclude that the CNM, which is corrected for the H I emission from PDRs, contributes $\sim 15 \%$ to the [C II] observed in the inner radius of $2 \mathrm{kpc}$. However, the CNM may contribute about $40 \%$ of the observed [C II] in the outer, northern disk, where $\mathrm{HI}$ emission is much stronger relative to $[\mathrm{C}$ II $]$.

Acknowledgements. We thank S. Hailey-Dunsheath and J.Gracia-Carpio for providing us data shown in Fig. 9. We thank an anonymous referee for insightful comments.

\section{References}

Bakes, E. L. O., \& Tielens, A. G. G. M. 1994, ApJ, 427, 822

Bolatto, A. D., Jackson, J. M., \& Ingalls, J. G. 1999, ApJ, 513, 275

Boquien, M., Calzetti, D., Combes, F., et al. 2011, AJ, 142, 111

Braine, J., Gratier, P., Kramer, C., et al. 2012, A\&A, 544, A55

Brauher, J. R., Dale, D. A., \& Helou, G. 2008, ApJS, 178, 280

Buchbender, C., Kramer, C., Gonzalez-Garcia, M., et al. 2013, A\&A, 549, A17

Cox, P., Krips, M., Neri, R., et al. 2011, ApJ, 740, 63

Crawford, M. K., Genzel, R., Townes, C. H., \& Watson, D. M. 1985, ApJ, 291, 755

Dale, D. A., \& Helou, G. 2002, ApJ, 576, 159

Dickey, J. M., Mebold, U., Stanimirovic, S., \& Staveley-Smith, L. 2000, ApJ, 536, 756

Engargiola, G., Plambeck, R. L., Rosolowsky, E., \& Blitz, L. 2003, ApJS, 149, 343

Freedman, W. L., Wilson, C. D., \& Madore, B. F. 1991, ApJ, 372, 455

Gardan, E., Braine, J., Schuster, K. F., Brouillet, N., \& Sievers, A. 2007, A\&A, 473, 91

Graciá-Carpio, J., Sturm, E., Hailey-Dunsheath, S., et al. 2011, ApJ, 728, L7

Gratier, P., Braine, J., Rodriguez-Fernandez, N. J., et al. 2010, A\&A, 522, A3

Gratier, P., Braine, J., Rodriguez-Fernandez, N. J., et al. 2012, A\&A, 542, A108

Gry, C., Swinyard, B., Harwood, A., et al. 2003, The ISO Handbook, Volume III - LWS - The Long Wavelength Spectrometer, eds. M. F. Kessler, et al.

Habart, E., Verstraete, L., Boulanger, F., et al. 2001, A\&A, 373, 702

Habing, H. J. 1968, Bull. Astron. Inst. Netherlands, 19, 421

Hailey-Dunsheath, S., Nikola, T., Stacey, G. J., et al. 2010, ApJ, 714, L162

Heiner, J. S., Allen, R. J., \& van der Kruit, P. C. 2011, MNRAS, 416, 2

Henry, R. B. C., Edmunds, M. G., \& Köppen, J. 2000, ApJ, 541, 660

Higdon, S. J. U., Higdon, J. L., van der Hulst, J. M., \& Stacey, G. J. 2003, ApJ, 592,161

Hollenbach, D. J., \& Tielens, A. G. G. M. 1997, ARA\&A, 35, 179

Hoopes, C. G., \& Walterbos, R. A. M. 2000, ApJ, 541, 597

Hunter, I., Dufton, P. L., Smartt, S. J., et al. 2007, A\&A, 466, 277

Israel, F. P., \& Maloney, P. R. 2011, A\&A, 531, A19

Israel, F. P., Maloney, P. R., Geis, N., et al. 1996, ApJ, 465, 738

Jakob, H., Kramer, C., Simon, R., et al. 2007, A\&A, 461, 999

Kaufman, M. J., Wolfire, M. G., Hollenbach, D. J., \& Luhman, M. L. 1999, ApJ, 527,795

Kaufman, M. J., Wolfire, M. G., \& Hollenbach, D. J. 2006, ApJ, 644, 283

Kessler, M. F., Steinz, J. A., Anderegg, M. E., et al. 1996, A\&A, 315, L27

Kramer, C., Buchbender, C., Xilouris, E. M., et al. 2010, A\&A, 518, L67

Langer, W. D., Velusamy, T., Pineda, J. L., et al. 2010, A\&A, 521, L17

Luhman, M. L., Satyapal, S., Fischer, J., et al. 2003, ApJ, 594, 758

Madden, S. C., Geis, N., Genzel, R., et al. 1993, ApJ, 407, 579

Madden, S. C., Poglitsch, A., Geis, N., Stacey, G. J., \& Townes, C. H. 1997, ApJ, 483, 200

Magrini, L., Stanghellini, L., Corbelli, E., Galli, D., \& Villaver, E. 2010, A\&A, 512, A63

Malhotra, S., Kaufman, M. J., Hollenbach, D., et al. 2001, ApJ, 561, 766

Mizutani, M., Onaka, T., \& Shibai, H. 2004, A\&A, 423, 579

Mookerjea, B., Kramer, C., Buchbender, C., et al. 2011, A\&A, 532, A152

Okada, Y., Pilleri, P., Berné, O., et al. 2013, A\&A, 553, A2

Putman, M. E., Peek, J. E. G., Muratov, A., et al. 2009, ApJ, 703, 1486

Rodriguez-Fernandez, N. J., Braine, J., Brouillet, N., \& Combes, F. 2006, A\&A, 453, 77 
C. Kramer et al.: Gas and dust cooling along the major axis of M33 (HerM33es)

Röllig, M., Ossenkopf, V., Jeyakumar, S., Stutzki, J., \& Sternberg, A. 2006, A\&A, 451, 917

Rubin, D., Hony, S., Madden, S. C., et al. 2009, A\&A, 494, 647

Sharma, S., Corbelli, E., Giovanardi, C., Hunt, L. K., \& Palla, F. 2011, A\&A, 534, A96

Sofia, U. J., Cardelli, J. A., Guerin, K. P., \& Meyer, D. M. 1997, ApJ, 482, L105

Stacey, G. J., Geis, N., Genzel, R., et al. 1991, ApJ, 373, 423

Stacey, G. J., Hailey-Dunsheath, S., Ferkinhoff, C., et al. 2010, ApJ, 724, 957

Sternberg, A. 1988, ApJ, 332, 400

Sturm, E., Bauer, O. H., Brauer, J., et al. 1998, in Astronomical Data Analysis Software and Systems VII, eds. R. Albrecht, R. N. Hook, \& H. A. Bushouse, ASP Conf. Ser., 145, 161
Swinyard, B. M., Burgdorf, M. J., Clegg, P. E., et al. 1998, in SPIE Conf. Ser. 3354, ed. A. M. Fowler, 888

Tabatabaei, F. S., Beck, R., Krause, M., et al. 2007, A\&A, 466, 509

Tielens, A. G. G. M., \& Hollenbach, D. 1985, ApJ, 291, 722

Vastel, C., Spaans, M., Ceccarelli, C., Tielens, A. G. G. M., \& Caux, E. 2001, A\&A, 376, 1064

Verley, S., Hunt, L. K., Corbelli, E., \& Giovanardi, C. 2007, A\&A, 476, 1161

Verley, S., Corbelli, E., Giovanardi, C., \& Hunt, L. K. 2009, A\&A, 493, 453

Weingartner, J. C., \& Draine, B. T. 2001, ApJS, 134, 263

Wolfire, M. G., Hollenbach, D., McKee, C. F., Tielens, A. G. G. M., \& Bakes, E. L. O. 1995, ApJ, 443, 152

Xilouris, E. M., Tabatabaei, F. S., Boquien, M., et al. 2012, A\&A, 543, A74

Pages 12 to 17 are available in the electronic edition of the journal at http: //www . aanda . org 


\section{Appendix A: List of positions}

Tables A.1 and A.2 list the 77 positions observed using ISO/LWS, their respective galacto-centric distance, and the detection of [C II].

\section{Appendix B: Intensities at the observed ISO/LWS positions}

The IRAM $30 \mathrm{~m} \mathrm{CO} \mathrm{2-1} \mathrm{data} \mathrm{on} \mathrm{the} T_{\mathrm{A}}^{*}$ scale were converted into $T_{\mathrm{mb}}$ temperatures using the forward efficiency $F_{\text {eff }}=0.90$ and the main beam efficiency $B_{\text {eff }}=0.49: T_{\mathrm{mb}}=\left(F_{\mathrm{eff}} / B_{\mathrm{eff}}\right) T_{A}^{*}$. The CO 2-1 map was first smoothed from the original 11" resolution to $22^{\prime \prime}$ using a Gaussian kernel. To derive CO 1-0 intensities from the CO 2-1 data, we used a linear function of the $2-1 / \mathrm{CO} 1-0$ ratio, which drops from 0.8 in the nucleus of M 33 to 0.5 at a galactocentric distance of $8.5 \mathrm{kpc}$ (Gratier et al. 2010). Next, we smoothed the map to the ISO/LWS resolution and converted the data to intensities in erg $\mathrm{cm}^{-2} \mathrm{~s}^{-1} \mathrm{sr}^{-1}$.

Both the $\mathrm{HI}$ data and the $\mathrm{H} \alpha$ data were also smoothed to the LWS resolution. The original resolutions of the $\mathrm{HI}$ data were $11^{\prime \prime}$, while we assumed an original pencil beam for the $\mathrm{H} \alpha$ data. Table B. 1 lists the resulting integrated intensities of [C II], HI, H $\alpha, \mathrm{CO} 2-1,1-0$, the FIR continuum, and the TIR/FIR ratio for all positions and stacked areas.

\section{Appendix C: Sample [CII] ISO/LWS spectra and SEDs}

We show here four examples of the [C II] spectra and SEDs obtained. Figure 1a shows the only spectrum with a baseline fit of order one. Figure 1c shows the spectrum from the nucleus, where the lowest rms and highest flux are reached. The highest rms and the lowest flux peak of the four spectra are shown in position N61 (Fig. 1g), which belongs to the northern, outer N2 region. The SEDs show the strongest warm dust component in the nucleus (Fig. 1d), while the weakest warm component is seen in N61 (Fig. 1h). Cold dust components are similar in the inter-arm region (Fig. 1b), the nucleus, and in BCLM302 (Fig. 1f). The outer points also show weaker cold dust emission than the other regions.

\section{Appendix D: [CII]/TIR vs. CO/TIR}

For completeness, we show the diagnostic plot of [C II] vs. CO luminosities in Fig. D.1, which are normalized with the TIR luminosities derived from the two-greybody fits described in Sect. 2.2. 
C. Kramer et al.: Gas and dust cooling along the major axis of M 33 (HerM33es)

Table A.1. ISO/LWS positions observed in [C II] along the southern part of the major axis of M33.

\begin{tabular}{|c|c|c|c|c|c|}
\hline $\begin{array}{l}\text { Pos ID } \\
\text { (1) }\end{array}$ & $\begin{array}{l}\text { RA } \\
(2)\end{array}$ & $\begin{array}{l}\text { Dec } \\
\text { (3) }\end{array}$ & $\begin{array}{l}\text { Ang. Dis ['] } \\
\text { (4) }\end{array}$ & $\begin{array}{l}\text { Lin. Dis }[\mathrm{kpc}] \\
(5)\end{array}$ & $\begin{array}{c}{[\mathrm{C} \text { II }] \text { detection? }} \\
(6)\end{array}$ \\
\hline S1 & $01: 32: 57$ & $+30: 10: 06$ & -32.70 & -7.99 & S1-S8 \\
\hline $\mathrm{S} 2$ & $01: 32: 58$ & $+30: 10: 52$ & -31.80 & -7.77 & $\mathrm{~S} 1-\mathrm{S} 8$ \\
\hline S3 & $01: 32: 59$ & $+30: 11: 38$ & -31.00 & -7.57 & S1-S8 \\
\hline S4 & 01:33:01 & $+30: 12: 24$ & -30.10 & -7.35 & $\mathrm{~S} 1-\mathrm{S} 8$ \\
\hline S5 & 01:33:02 & $+30: 13: 10$ & -29.30 & -7.16 & $\mathrm{~S} 1-\mathrm{S} 8$ \\
\hline S6 & 01:33:03 & $+30: 13: 56$ & -28.40 & -6.94 & $\mathrm{~S} 1-\mathrm{S} 8$ \\
\hline S7 & 01:33:05 & $+30: 14: 42$ & -27.50 & -6.72 & S1-S8 \\
\hline S8 & 01:33:06 & $+30: 15: 28$ & -26.70 & -6.52 & S1-S8 \\
\hline S9 & 01:33:07 & $+30: 16: 14$ & -25.80 & -6.30 & S9-S14 \\
\hline S10 & 01:33:08 & $+30: 17: 00$ & -25.00 & -6.11 & S9-S14 \\
\hline S11 & 01:33:10 & $+30: 17: 46$ & -24.10 & -5.89 & S9-S14 \\
\hline S12 & 01:33:11 & $+30: 18: 32$ & -23.30 & -5.69 & S9-S14 \\
\hline S13 & $01: 33: 12$ & $+30: 19: 18$ & -22.40 & -5.47 & S9-S14 \\
\hline S14 & $01: 33: 14$ & $+30: 20: 04$ & -21.60 & -5.28 & S9-S14 \\
\hline S15 & $01: 33: 15$ & $+30: 20: 50$ & -20.70 & -5.06 & S15-S19 \\
\hline S16 & $01: 33: 16$ & $+30: 21: 36$ & -19.90 & -4.86 & S15-S19 \\
\hline S17 & $01: 33: 18$ & $+30: 22: 22$ & -19.00 & -4.64 & S15-S19 \\
\hline S18 & $01: 33: 19$ & $+30: 23: 08$ & -18.10 & -4.42 & S15-S19 \\
\hline S19 & $01: 33: 20$ & $+30: 23: 54$ & -17.30 & -4.23 & S15-S19 \\
\hline S20 & $01: 33: 24$ & $+30: 24: 40$ & -16.40 & -4.01 & S20-S21 \\
\hline S21 & $01: 33: 25$ & $+30: 25: 27$ & -15.60 & -3.81 & S20-S21 \\
\hline S22 & $01: 33: 27$ & $+30: 26: 13$ & -14.70 & -3.59 & $\mathrm{~S} 22-\mathrm{S} 23$ \\
\hline S23 & $01: 33: 28$ & $+30: 26: 59$ & -13.90 & -3.40 & S22-S23 \\
\hline S24 & $01: 33: 30$ & $+30: 27: 45$ & -13.00 & -3.18 & S24-S26 \\
\hline S25 & $01: 33: 31$ & $+30: 28: 31$ & -12.20 & -2.98 & S24-S26 \\
\hline S26 & $01: 33: 33$ & $+30: 29: 17$ & -11.30 & -2.76 & S24-S26 \\
\hline S27 & $01: 33: 34$ & $+30: 30: 03$ & -10.50 & -2.57 & $\mathrm{X}$ \\
\hline S28 & $01: 33: 36$ & $+30: 30: 49$ & -9.60 & -2.35 & $X$ \\
\hline S29 & $01: 33: 37$ & $+30: 31: 35$ & -8.75 & -2.14 & $X$ \\
\hline S30 & 01:33:39 & $+30: 32: 21$ & -7.89 & -1.93 & $X$ \\
\hline S31 & $01: 33: 40$ & $+30: 33: 07$ & -7.04 & -1.72 & $X$ \\
\hline S32 & $01: 33: 42$ & $+30: 33: 53$ & -6.19 & -1.51 & $X$ \\
\hline S33 & $01: 33: 43$ & $+30: 34: 39$ & -5.33 & -1.30 & $X$ \\
\hline S34 & $01: 33: 45$ & $+30: 35: 25$ & -4.48 & -1.09 & $X$ \\
\hline S35 & $01: 33: 46$ & $+30: 36: 11$ & -3.63 & -0.89 & $X$ \\
\hline S36 & 01:33:48 & $+30: 36: 57$ & -2.79 & -0.68 & $X$ \\
\hline S37 & $01: 33: 49$ & $+30: 37: 43$ & -1.95 & -0.48 & $X$ \\
\hline S38 & 01:33:51 & $+30: 38: 29$ & -1.14 & -0.28 & $X$ \\
\hline
\end{tabular}

Notes. Columns (4) and (5) list galacto-centric distances. Negative values indicate southern positions. Column (6) indicates whether [C II] emission was detected above $3 \sigma$ at a given position (marked by "X") or were averaged over a given range of positions. 
A\&A 553, A114 (2013)

Table A.2. ISO/LWS positions observed in [C II] along the northern part of the major axis of M 33 .

\begin{tabular}{|c|c|c|c|c|c|}
\hline $\begin{array}{l}\text { Pos ID } \\
\text { (1) }\end{array}$ & $\begin{array}{c}\mathrm{RA}(\mathrm{J} 2000) \\
(2)\end{array}$ & $\begin{array}{c}\operatorname{Dec}(\mathrm{J} 2000) \\
(3)\end{array}$ & $\begin{array}{l}\text { Ang. Dis ['] } \\
\text { (4) }\end{array}$ & $\begin{array}{l}\text { Lin. Dis }[\mathrm{kpc}] \\
\text { (5) }\end{array}$ & $\begin{array}{c}\text { [C II] Detection? } \\
\text { (6) }\end{array}$ \\
\hline 39 (Nucleus) & $01: 33: 51$ & $+30: 39: 37$ & 0.00 & 0.00 & $\mathrm{X}$ \\
\hline N40 & $01: 33: 54$ & $+30: 40: 01$ & .80 & 0.20 & $X$ \\
\hline N41 & $01: 33: 55$ & $+30: 40: 47$ & 1.58 & 0.39 & $X$ \\
\hline N42 & 01:33:57 & $+30: 41: 33$ & 2.42 & 0.59 & $X$ \\
\hline N43 & 01:33:58 & $+30: 42: 19$ & 3.26 & 0.80 & $X$ \\
\hline N44 & 01:34:00 & $+30: 43: 05$ & 4.11 & 1.00 & $X$ \\
\hline N45 & 01:34:01 & $+30: 43: 51$ & 4.96 & 1.21 & $X$ \\
\hline N46 & 01:34:03 & $+30: 44: 37$ & 5.81 & 1.42 & $X$ \\
\hline N47 & 01:34:04 & $+30: 45: 23$ & 6.67 & 1.63 & $X$ \\
\hline N48 & 01:34:06 & $+30: 46: 09$ & 7.52 & 1.84 & $X$ \\
\hline N49 & 01:34:07 & $+30: 46: 55$ & 8.38 & 2.05 & $\mathrm{X}$ \\
\hline N50 & 01:34:09 & $+30: 47: 41$ & 9.23 & 2.26 & $X$ \\
\hline N51 & 01:34:10 & $+30: 48: 27$ & 10.1 & 2.47 & $X$ \\
\hline N52 & 01:34:12 & $+30: 49: 13$ & 10.9 & 2.66 & $X$ \\
\hline N53 & $01: 34: 13$ & $+30: 49: 59$ & 11.8 & 2.88 & $X$ \\
\hline N54 & $01: 34: 15$ & $+30: 50: 45$ & 12.6 & 3.08 & $X$ \\
\hline N55 & $01: 34: 16$ & $+30: 51: 31$ & 13.5 & 3.30 & $X$ \\
\hline N56 & $01: 34: 18$ & $+30: 52: 17$ & 14.4 & 3.52 & $\mathrm{X}$ \\
\hline N57 & 01:34:19 & $+30: 53: 03$ & 15.2 & 3.71 & N57-N60 \\
\hline N58 & 01:34:21 & $+30: 53: 49$ & 16.1 & 3.93 & N57-N60 \\
\hline N59 & $01: 34: 23$ & $+30: 54: 35$ & 16.9 & 4.13 & N57-N60 \\
\hline N60 & $01: 34: 24$ & $+30: 55: 21$ & 17.8 & 4.35 & N57-N60 \\
\hline N61 & $01: 34: 26$ & $+30: 56: 07$ & 18.7 & 4.57 & $X$ \\
\hline N62 & $01: 34: 27$ & $+30: 56: 53$ & 19.5 & 4.76 & $X$ \\
\hline N63 & 01:34:29 & $+30: 57: 39$ & 20.4 & 4.98 & $X$ \\
\hline N64 & 01:34:30 & $+30: 58: 25$ & 21.2 & 5.18 & - \\
\hline N65 & $01: 34: 32$ & $+30: 59: 11$ & 22.1 & 5.40 & $X$ \\
\hline N66 & $01: 34: 33$ & $+30: 59: 57$ & 22.9 & 5.60 & $X$ \\
\hline N67 & 01:34:35 & $+31: 00: 43$ & 23.8 & 5.82 & N67-N68 \\
\hline N68 & 01:34:36 & $+31: 01: 29$ & 24.6 & 6.01 & N67-N68 \\
\hline N69 & $01: 34: 38$ & $+31: 02: 15$ & 25.5 & 6.23 & $X$ \\
\hline N70 & 01:34:39 & $+31: 03: 01$ & 26.4 & 6.45 & N70-N72 \\
\hline N71 & 01:34:41 & $+31: 03: 47$ & 27.2 & 6.65 & N70-N72 \\
\hline N72 & $01: 34: 42$ & $+31: 04: 33$ & 28.1 & 6.87 & N70-N72 \\
\hline N73 & 01:34:44 & $+31: 05: 19$ & 28.9 & 7.06 & N73-N77 \\
\hline N74 & $01: 34: 45$ & $+31: 06: 05$ & 29.8 & 7.28 & N73-N77 \\
\hline N75 & $01: 34: 47$ & $+31: 06: 51$ & 30.6 & 7.48 & N73-N77 \\
\hline N76 & $01: 34: 48$ & $+31: 07: 38$ & 31.5 & 7.70 & N73-N77 \\
\hline N77 & $01: 34: 50$ & $+31: 08: 24$ & 32.3 & 7.89 & N73-N77 \\
\hline
\end{tabular}

Notes. Columns (4) and (5) list galacto-centric distances. Column (6) indicates whether [C II] emission was detected above $3 \sigma$ at a given position (marked by "X"), where the emission was averaged over a given range of positions, or whether no emission was detected (marked by "-"). 
C. Kramer et al.: Gas and dust cooling along the major axis of M 33 (HerM33es)

Table B.1. Integrated intensities at the positions with [C II] detections.

\begin{tabular}{|c|c|c|c|c|c|c|c|}
\hline 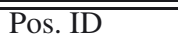 & $\overline{\text { [C II }]}$ & $\overline{\overline{\mathrm{HI}}}$ & $\overline{\overline{\mathrm{H} \alpha}}$ & $\overline{\mathrm{CO}(2-1)}$ & $=\mathrm{CO} \mathrm{1-0}$ & "FIR & "TIR/FIR \\
\hline S1-S8 & $2.20 \mathrm{e}-06$ & $6.88 \mathrm{e}-13$ & $3.05 e-07$ & - & - & $9.12 \mathrm{e}-05$ & $2.52 \mathrm{e}+00$ \\
\hline S9-S14 & $1.28 \mathrm{e}-06$ & $1.37 \mathrm{e}-13$ & 3.11e-07 & $1.18 \mathrm{e}-09$ & $8.32 \mathrm{e}-11$ & $4.25 \mathrm{e}-05$ & $2.50 \mathrm{e}+00$ \\
\hline S15-S19 & $1.26 \mathrm{e}-06$ & $1.73 \mathrm{e}-12$ & $8.68 \mathrm{e}-07$ & $3.13 e-09$ & $2.39 \mathrm{e}-10$ & $4.17 \mathrm{e}-05$ & $2.47 \mathrm{e}+00$ \\
\hline $\mathrm{S} 20-\mathrm{S} 21$ & $4.70 \mathrm{e}-06$ & $1.48 \mathrm{e}-12$ & $3.75 e-06$ & $6.35 e-09$ & $5.15 \mathrm{e}-10$ & $8.39 \mathrm{e}-04$ & $1.54 \mathrm{e}+00$ \\
\hline S22-S23 & $3.75 \mathrm{e}-06$ & $1.86 \mathrm{e}-12$ & $1.20 \mathrm{e}-06$ & $7.06 \mathrm{e}-09$ & $5.87 \mathrm{e}-10$ & $4.61 \mathrm{e}-04$ & $1.89 \mathrm{e}+00$ \\
\hline S24-S26 & $2.69 \mathrm{e}-06$ & $2.00 \mathrm{e}-13$ & $9.64 \mathrm{e}-07$ & $1.70 \mathrm{e}-09$ & $1.44 \mathrm{e}-10$ & $2.62 \mathrm{e}-04$ & $2.32 \mathrm{e}+00$ \\
\hline S27 & $5.04 \mathrm{e}-06$ & $7.98 \mathrm{e}-13$ & $3.63 \mathrm{e}-06$ & $3.10 \mathrm{e}-09$ & $5.51 \mathrm{e}-10$ & $4.82 \mathrm{e}-04$ & $1.44 \mathrm{e}+00$ \\
\hline S28 & $8.38 \mathrm{e}-06$ & $1.08 \mathrm{e}-12$ & $7.35 \mathrm{e}-06$ & $5.92 \mathrm{e}-09$ & $1.04 \mathrm{e}-09$ & $8.19 \mathrm{e}-04$ & $1.48 \mathrm{e}+00$ \\
\hline S29 & $1.42 \mathrm{e}-05$ & $2.11 \mathrm{e}-12$ & $1.34 \mathrm{e}-05$ & $1.69 \mathrm{e}-08$ & $2.94 \mathrm{e}-09$ & $1.73 \mathrm{e}-03$ & $1.49 \mathrm{e}+00$ \\
\hline S30 & $1.57 \mathrm{e}-05$ & $2.02 \mathrm{e}-12$ & $1.61 \mathrm{e}-05$ & $1.81 \mathrm{e}-08$ & $3.11 \mathrm{e}-09$ & $1.72 \mathrm{e}-03$ & $1.73 e+00$ \\
\hline S31 & $1.45 \mathrm{e}-05$ & $1.68 \mathrm{e}-12$ & $1.24 \mathrm{e}-05$ & $1.28 \mathrm{e}-08$ & $2.17 \mathrm{e}-09$ & $1.62 \mathrm{e}-03$ & $1.56 \mathrm{e}+00$ \\
\hline S32 & $6.97 \mathrm{e}-06$ & $1.35 \mathrm{e}-12$ & $7.45 \mathrm{e}-06$ & $9.68 \mathrm{e}-09$ & $1.63 \mathrm{e}-09$ & $1.34 \mathrm{e}-03$ & $1.38 \mathrm{e}+00$ \\
\hline S33 & $9.54 \mathrm{e}-06$ & $1.25 \mathrm{e}-12$ & $5.81 \mathrm{e}-06$ & $8.88 \mathrm{e}-09$ & $1.48 \mathrm{e}-09$ & $1.16 \mathrm{e}-03$ & $1.44 \mathrm{e}+00$ \\
\hline S34 & $1.43 e-05$ & $1.74 \mathrm{e}-12$ & $9.92 \mathrm{e}-06$ & $1.41 \mathrm{e}-08$ & $2.32 \mathrm{e}-09$ & $1.78 \mathrm{e}-03$ & $1.42 \mathrm{e}+00$ \\
\hline S35 & $1.78 \mathrm{e}-05$ & $2.08 \mathrm{e}-12$ & $1.66 \mathrm{e}-05$ & $2.75 e-08$ & $4.30 \mathrm{e}-09$ & $2.00 \mathrm{e}-03$ & $1.32 \mathrm{e}+00$ \\
\hline S36 & $1.64 \mathrm{e}-05$ & $1.55 \mathrm{e}-12$ & $1.41 \mathrm{e}-05$ & $2.02 \mathrm{e}-08$ & $3.15 \mathrm{e}-09$ & $2.01 \mathrm{e}-03$ & $1.39 \mathrm{e}+00$ \\
\hline S37 & $1.41 \mathrm{e}-05$ & $1.02 \mathrm{e}-12$ & $1.38 \mathrm{e}-05$ & $1.50 \mathrm{e}-08$ & $2.34 \mathrm{e}-09$ & $1.85 \mathrm{e}-03$ & $1.37 \mathrm{e}+00$ \\
\hline S38 & $1.75 \mathrm{e}-05$ & $1.04 \mathrm{e}-12$ & $1.90 \mathrm{e}-05$ & $1.75 e-08$ & $2.74 \mathrm{e}-09$ & $2.25 \mathrm{e}-03$ & $1.34 \mathrm{e}+00$ \\
\hline 39 (Nucleus) & $3.27 e-05$ & $1.92 \mathrm{e}-12$ & $2.77 e-05$ & $3.41 \mathrm{e}-08$ & $5.34 \mathrm{e}-09$ & $3.62 \mathrm{e}-03$ & $1.34 \mathrm{e}+00$ \\
\hline $\mathrm{N} 40$ & $2.36 \mathrm{e}-05$ & $2.01 \mathrm{e}-12$ & $1.52 \mathrm{e}-05$ & $2.85 e-08$ & $4.45 \mathrm{e}-09$ & $2.78 \mathrm{e}-03$ & $1.33 \mathrm{e}+00$ \\
\hline N41 & $1.45 \mathrm{e}-05$ & $2.28 \mathrm{e}-12$ & $1.03 e-05$ & $3.17 \mathrm{e}-08$ & $4.96 \mathrm{e}-09$ & $2.45 \mathrm{e}-03$ & $1.39 \mathrm{e}+00$ \\
\hline N42 & $1.34 \mathrm{e}-05$ & $2.09 \mathrm{e}-12$ & $8.94 \mathrm{e}-06$ & $3.72 \mathrm{e}-08$ & $5.81 \mathrm{e}-09$ & $2.16 \mathrm{e}-03$ & $1.40 \mathrm{e}+00$ \\
\hline N43 & $1.03 e-05$ & $1.64 \mathrm{e}-12$ & $9.89 \mathrm{e}-06$ & $2.65 e-08$ & $4.14 \mathrm{e}-09$ & $1.33 \mathrm{e}-03$ & $1.69 \mathrm{e}+00$ \\
\hline N44 & $7.00 \mathrm{e}-06$ & $1.05 \mathrm{e}-12$ & $4.74 \mathrm{e}-06$ & $1.38 \mathrm{e}-08$ & $2.27 \mathrm{e}-09$ & $8.56 \mathrm{e}-04$ & $1.47 \mathrm{e}+00$ \\
\hline N45 & $8.27 \mathrm{e}-06$ & $1.36 \mathrm{e}-12$ & $5.99 \mathrm{e}-06$ & $1.58 \mathrm{e}-08$ & $2.62 \mathrm{e}-09$ & $8.19 \mathrm{e}-04$ & $1.73 e+00$ \\
\hline N46 & $6.15 \mathrm{e}-06$ & $1.21 \mathrm{e}-12$ & $4.08 \mathrm{e}-06$ & $1.11 \mathrm{e}-08$ & $1.85 \mathrm{e}-09$ & $6.41 \mathrm{e}-04$ & $1.68 \mathrm{e}+00$ \\
\hline N47 & $5.77 \mathrm{e}-06$ & $1.02 \mathrm{e}-12$ & $2.85 \mathrm{e}-06$ & $1.10 \mathrm{e}-08$ & $1.86 \mathrm{e}-09$ & $5.28 \mathrm{e}-04$ & $1.63 e+00$ \\
\hline $\mathrm{N} 48$ & $8.00 \mathrm{e}-06$ & $1.95 \mathrm{e}-12$ & $4.71 \mathrm{e}-06$ & $1.83 \mathrm{e}-08$ & $3.13 \mathrm{e}-09$ & $8.83 e-04$ & $1.48 \mathrm{e}+00$ \\
\hline N49 & $1.28 \mathrm{e}-05$ & $2.77 \mathrm{e}-12$ & $1.56 \mathrm{e}-05$ & $2.17 \mathrm{e}-08$ & $3.75 e-09$ & $1.79 \mathrm{e}-03$ & $1.43 \mathrm{e}+00$ \\
\hline N50 & $1.49 \mathrm{e}-05$ & $2.69 \mathrm{e}-12$ & $1.41 \mathrm{e}-05$ & $2.39 \mathrm{e}-08$ & $4.18 \mathrm{e}-09$ & $1.30 \mathrm{e}-03$ & $1.89 \mathrm{e}+00$ \\
\hline N51 & $9.91 \mathrm{e}-06$ & $3.54 \mathrm{e}-12$ & $5.56 \mathrm{e}-06$ & $3.73 e-08$ & $6.59 \mathrm{e}-09$ & $1.01 \mathrm{e}-03$ & $1.96 \mathrm{e}+00$ \\
\hline N52 & $6.98 \mathrm{e}-06$ & $3.22 \mathrm{e}-12$ & $2.96 \mathrm{e}-06$ & $3.51 \mathrm{e}-08$ & $6.27 \mathrm{e}-09$ & $8.13 e-04$ & $1.83 \mathrm{e}+00$ \\
\hline N53 & $4.48 \mathrm{e}-06$ & $1.31 \mathrm{e}-12$ & $1.37 \mathrm{e}-06$ & $1.41 \mathrm{e}-08$ & $2.56 \mathrm{e}-09$ & $4.78 \mathrm{e}-04$ & $1.42 \mathrm{e}+00$ \\
\hline N54 & $3.72 \mathrm{e}-06$ & $1.12 \mathrm{e}-12$ & $2.21 \mathrm{e}-06$ & $6.24 \mathrm{e}-09$ & $1.14 \mathrm{e}-09$ & $3.22 \mathrm{e}-04$ & $1.78 \mathrm{e}+00$ \\
\hline N55 & $8.24 \mathrm{e}-06$ & $1.76 \mathrm{e}-12$ & $1.43 e-05$ & $1.34 \mathrm{e}-08$ & $2.48 \mathrm{e}-09$ & $8.23 e-04$ & $1.72 \mathrm{e}+00$ \\
\hline N56 & $5.23 \mathrm{e}-06$ & $1.96 \mathrm{e}-12$ & $1.26 \mathrm{e}-05$ & $1.50 \mathrm{e}-08$ & $2.80 \mathrm{e}-09$ & 7.96e-04 & $1.69 \mathrm{e}+00$ \\
\hline N57-N60 & $2.70 \mathrm{e}-06$ & $9.94 \mathrm{e}-13$ & $2.69 \mathrm{e}-06$ & $2.27 \mathrm{e}-09$ & $1.87 \mathrm{e}-10$ & $5.12 \mathrm{e}-04$ & $1.56 \mathrm{e}+00$ \\
\hline N61 & $4.13 e-06$ & $1.40 \mathrm{e}-12$ & $2.26 \mathrm{e}-06$ & $2.55 \mathrm{e}-09$ & $5.07 \mathrm{e}-10$ & $2.19 \mathrm{e}-04$ & $1.90 \mathrm{e}+00$ \\
\hline N62 & $4.71 \mathrm{e}-06$ & $2.54 \mathrm{e}-12$ & $5.81 \mathrm{e}-06$ & $4.41 \mathrm{e}-09$ & $8.87 \mathrm{e}-10$ & $4.09 \mathrm{e}-04$ & $1.74 \mathrm{e}+00$ \\
\hline N63 & $8.22 \mathrm{e}-06$ & $2.20 \mathrm{e}-12$ & $8.17 \mathrm{e}-06$ & $3.11 \mathrm{e}-09$ & $6.35 \mathrm{e}-10$ & $5.66 \mathrm{e}-04$ & $1.46 \mathrm{e}+00$ \\
\hline N65 & $2.38 \mathrm{e}-06$ & $9.94 \mathrm{e}-13$ & $1.26 \mathrm{e}-06$ & $3.57 \mathrm{e}-10$ & $7.48 \mathrm{e}-11$ & $1.47 \mathrm{e}-04$ & $1.75 \mathrm{e}+00$ \\
\hline N66 & $4.10 \mathrm{e}-06$ & $8.42 \mathrm{e}-13$ & $8.15 \mathrm{e}-06$ & $3.86 \mathrm{e}-10$ & $9.97 \mathrm{e}-11$ & $1.68 \mathrm{e}-04$ & $1.71 \mathrm{e}+00$ \\
\hline N67-N68 & $2.72 \mathrm{e}-06$ & $1.34 \mathrm{e}-12$ & $4.44 \mathrm{e}-06$ & $4.26 \mathrm{e}-10$ & $3.10 \mathrm{e}-10$ & $1.89 \mathrm{e}-04$ & $2.70 \mathrm{e}+00$ \\
\hline N69 & $2.76 \mathrm{e}-06$ & $1.20 \mathrm{e}-12$ & $1.31 \mathrm{e}-06$ & $4.89 \mathrm{e}-09$ & $3.45 \mathrm{e}-10$ & $8.09 \mathrm{e}-05$ & $2.08 \mathrm{e}+00$ \\
\hline N70-N72 & $1.19 \mathrm{e}-06$ & $7.98 \mathrm{e}-13$ & $3.19 \mathrm{e}-07$ & $1.45 e-09$ & $1.01 \mathrm{e}-10$ & $5.06 \mathrm{e}-05$ & $2.12 \mathrm{e}+00$ \\
\hline N73-N77 & $3.53 e-06$ & $1.97 \mathrm{e}-12$ & $5.25 \mathrm{e}-07$ & $4.24 \mathrm{e}-10$ & $2.83 \mathrm{e}-11$ & $8.51 \mathrm{e}-04$ & $2.31 \mathrm{e}+00$ \\
\hline
\end{tabular}

Notes. Intensities are given in $\mathrm{erg} \mathrm{s}^{-1} \mathrm{sr}^{-1} \mathrm{~cm}^{-2}$ at the angular resolution of the [C II] data. Values in bold face are upper limits. 


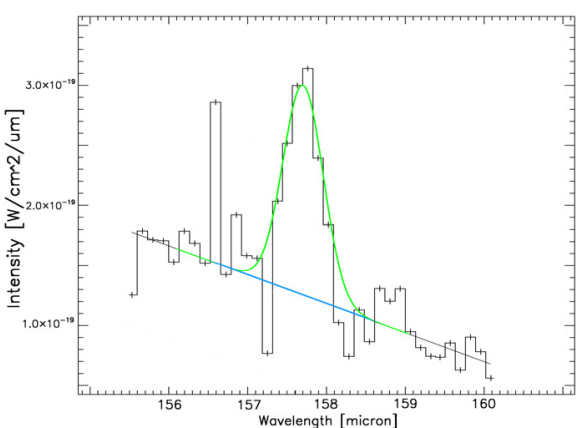

(a)

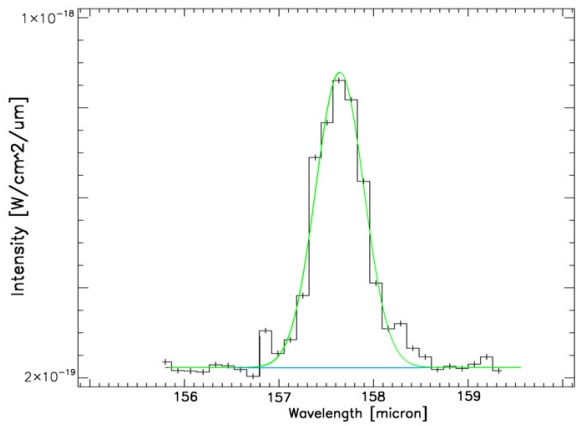

(c)

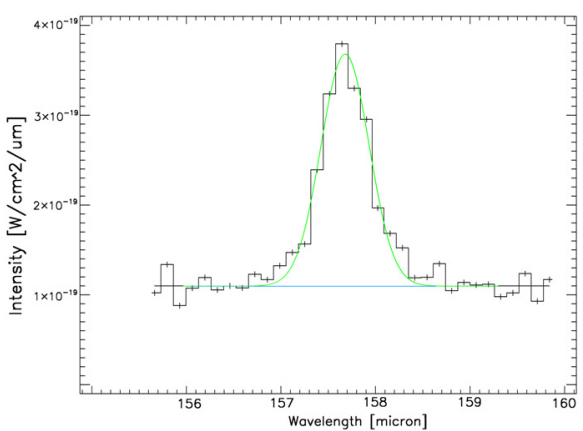

(e)

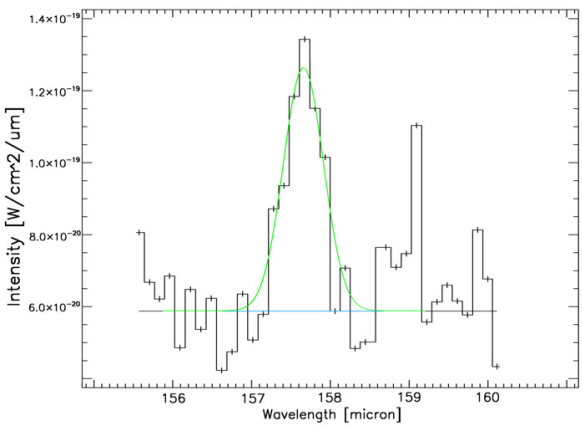

(g)

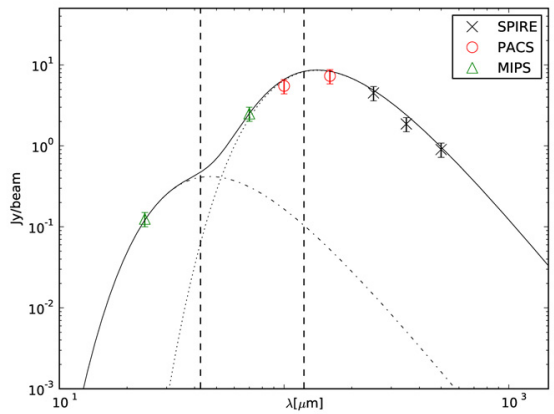

(b)

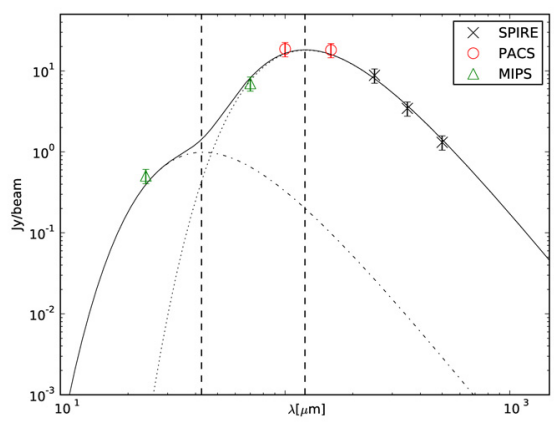

(d)

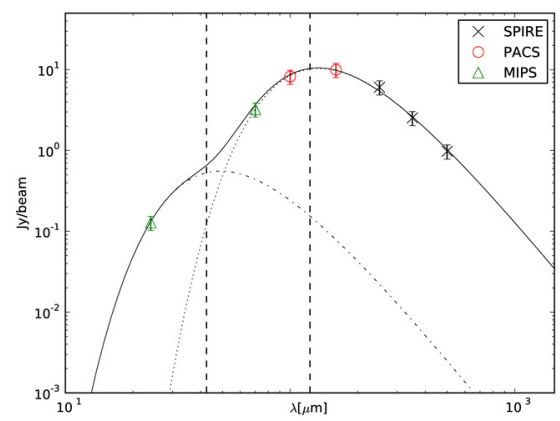

(f)

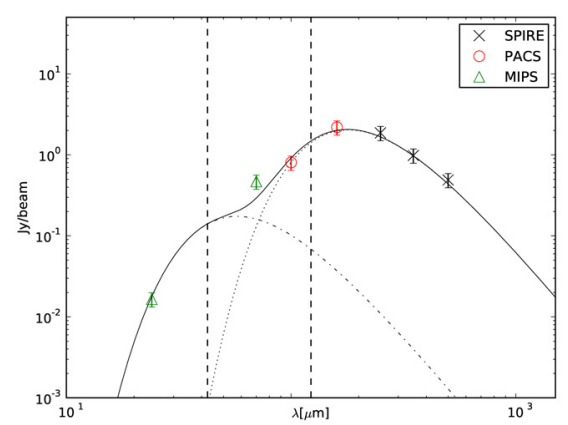

(h)

Fig. C.1. Examples of some of the [C II] ISO/LWS spectra and SEDs obtained in this work. The vertical dashed lines mark the integration interval to derive the FIR continuum, $42.5 \mu \mathrm{m}$ and $122.5 \mu \mathrm{m}$ (Dale \& Helou 2002). a), b) The first row shows the [C II] spectrum and the SED of the inter-arm position S32. c, d) The second row shows the spectrum and SED from the nucleus of M33. e), f) The third row shows observations of the H II region BCLMP 302 (N49). g), h) Finally, the last row shows the spectrum and the SED from one of the positions (N61) in the outer, southern N2 region. The SEDs show MIPS $24 \mu \mathrm{m}$ and $70 \mu \mathrm{m}$, PACS $100 \mu \mathrm{m}$ and $160 \mu \mathrm{m}$ and SPIRE $250 \mu \mathrm{m}, 350 \mu \mathrm{m}$ and $500 \mu \mathrm{m}$ data. In the [C II] spectra, green lines show Gaussian fits to the spectra and blue lines show fitted baselines. 
C. Kramer et al.: Gas and dust cooling along the major axis of M33 (HerM33es)

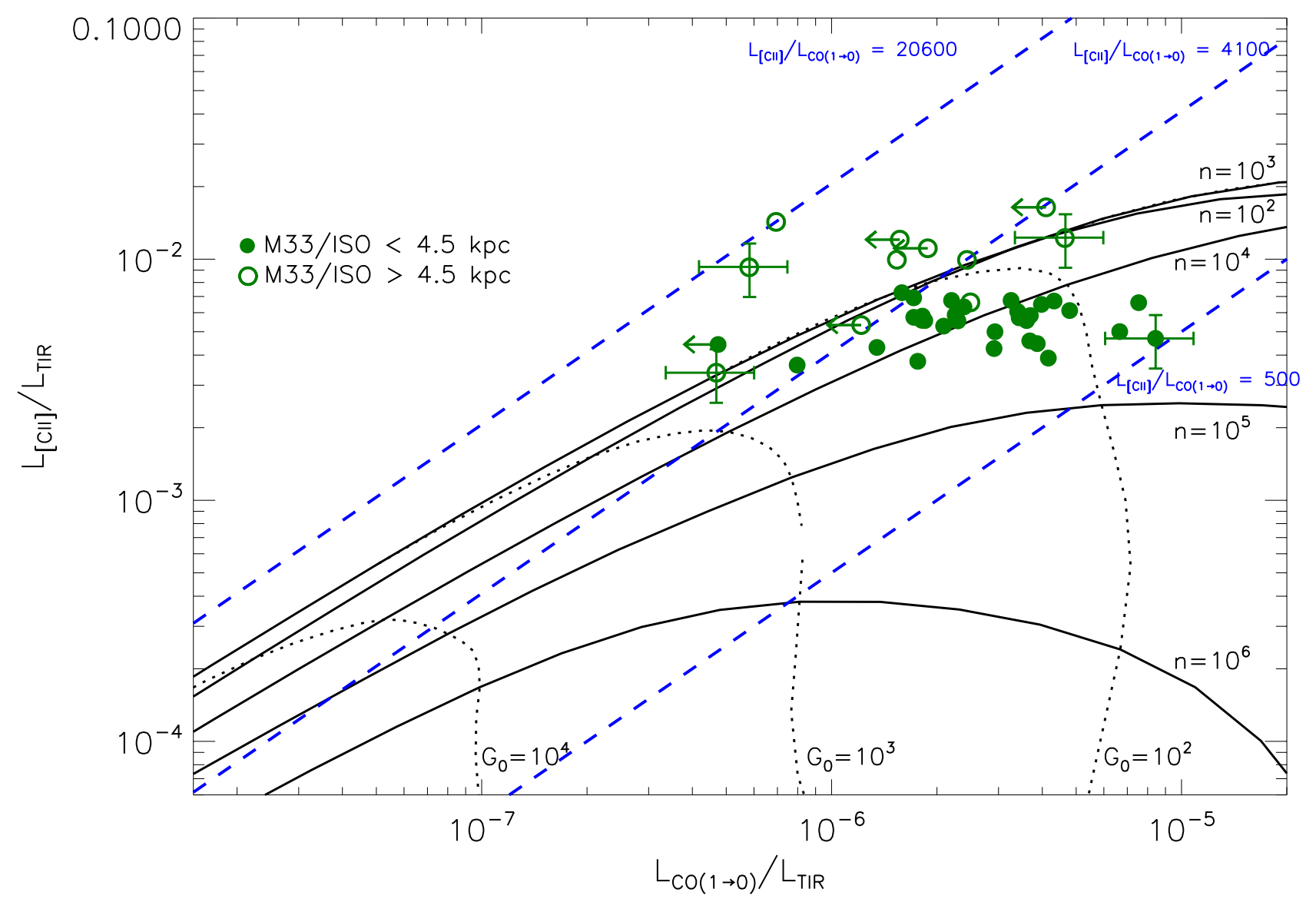

Fig. D.1. [C II] versus CO, which is normalized using the total infrared continuum (TIR). Big green filled circles show ISO/LWS data of the inner S1, N1 regions of M33, while open circles show data of the outer S2, N2 regions. The lowest [C II]/CO ratio observed with ISO/LWS in M 33 is 1000 (lower blue dashed line), while the highest ratio is 41200 (upper blue dashed line). Black solid and dotted lines indicate lines of constant density $n$ and FUV field $G_{0}$, respectively, from the standard K99 PDR model with $A_{\mathrm{V}}=10$ mag and solar metallicity $Z=1$. 